\title{
THE LYMAN ALPHA FOREST IN THE SPECTRA OF QUASISTELLAR OBJECTS
}

\author{
Michael Rauch \\ Astronomy Department, California Institute of Technology, Pasadena, \\ California 91125; e-mail: mr@astro.caltech.edu \\ KEY WORDS: quasistellar object (QSO) absorption lines, intergalactic medium (IGM), galaxy \\ formation, cosmology, ultraviolet background radiation
}

\begin{abstract}
Observations of redshifted Lyman $\alpha$ ( $\operatorname{Ly} \alpha)$ forest absorption in the spectra of quasistellar objects (QSOs) provide a highly sensitive probe of the distribution of gaseous matter in the universe. Over the past two decades, optical spectroscopy with large ground-based telescopes, and more recently ultraviolet (UV) spectroscopy from space, have yielded a wealth of information on what appears to be a gaseous, photoionized intergalactic medium (IGM), partly enriched by the products of stellar nucleosynthesis, residing in coherent structures over many hundreds of kiloparsecs.

Recent progress with cosmological hydro-simulations based on hierarchical structure formation models has led to important insights into the physical structures giving rise to the forest. If these ideas are correct, a truly inter- and protogalactic medium [at high redshift $(z \sim 3)$, the main repository of baryons] collapses under the influence of dark matter gravity into flattened or filamentary structures, which are seen in absorption against background QSOs. With decreasing redshift, galaxies forming in the denser regions may contribute an increasing part of the Ly $\alpha$ absorption cross section. Comparisons between large data samples from the new generation of telescopes and artificial Ly $\alpha$ forest spectra from cosmological simulations promise to become a useful cosmological tool.
\end{abstract}




\section{INTRODUCTION}

The Lyman $\alpha(\operatorname{Ly} \alpha)$ forest is an absorption phenomenon in the spectra of background quasistellar objects (QSOs). It can be observed in the ultraviolet (UV) and optical wavelength range, from the local universe up to the highest redshifts where QSOs are found (currently $z \sim 5$ ). Neutral hydrogen intersected by the line of sight (LOS) to a QSO will cause absorption of the QSO continuum by the redshifted Ly $\alpha$ (1215.67 $\AA$ ) UV resonance line. In an expanding universe homogeneously filled with gas, the continuously redshifted Ly $\alpha$ line will produce an absorption trough blueward of the QSO's Ly $\alpha$ emission line (independent predictions by Gunn \& Peterson 1965, Scheuer 1965, Shklovski 1965). Gunn \& Peterson found such a spectral region of reduced flux and used this measurement to put upper limits on the amount of intergalactic neutral hydrogen. The large cross section for the Ly $\alpha$ transition makes this technique by far the most sensitive method for detecting baryons at any redshift.

Bahcall \& Salpeter (1965) suggested that there should also be a population of discrete absorption lines from a more clumpy gas distribution, specifically from intervening groups of galaxies. Discrete lines were observed shortly thereafter (Lynds \& Stockton 1966, Burbidge et al 1966, Stockton \& Lynds 1966, Kinman 1966), but the quest for their precise origin has given rise to a long and, at times, controversial debate; only in recent years does the issue appear to have been resolved (see below). Soon thereafter, the simultaneous detection of higher order lines of the Lyman series (e.g. Baldwin et al 1974) had confirmed the suggestion (Lynds 1970) that most of the absorption is indeed from H I Ly $\alpha$. At higher spectral resolution, the Ly $\alpha$ forest can be resolved into hundreds (in $z>2$ QSO spectra) of distinct absorption lines, the appearance of which gave rise to the label Ly $\alpha$ forest (Weymann et al 1981; see Figure 1). A small fraction of the lines hidden in the forest are not caused by H I but belong to UV transitions

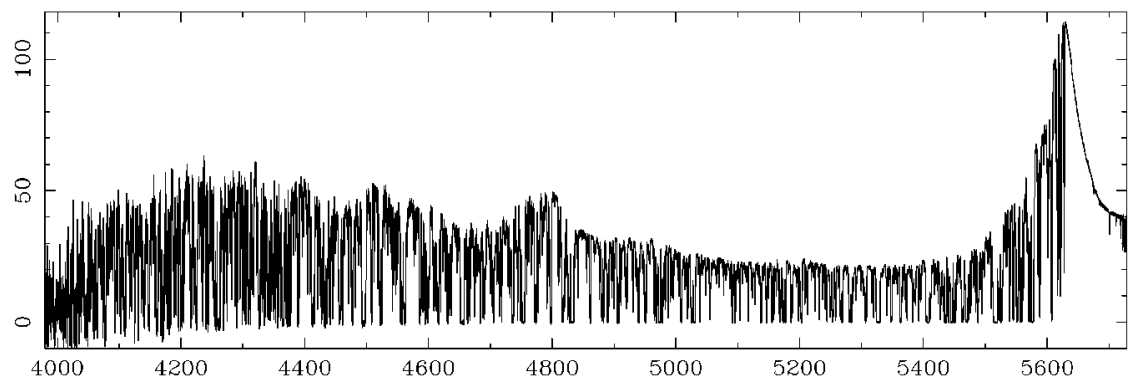

Figure 1 High resolution [full width at half maximum $(\mathrm{FWHM}) \approx 6.6 \mathrm{~km} \mathrm{~s}^{-1}$ ] spectrum of the $z_{\text {em }}=3.62$ QSO1422+23 $(V=16.5)$, taken with the Keck High Resolution Spectrograph (HIRES) (signal-to-noise ratio $\sim 150$ per resolution element, exposure time 25,000 s). Data from Womble et al (1996). 
from several common metal or heavy element ions (various ionization stages of $\mathrm{C}, \mathrm{O}, \mathrm{Mg}, \mathrm{Si}, \mathrm{Fe}$, and $\mathrm{Al}$ are most frequently seen). These metal lines are invariably associated with strong Ly $\alpha$ lines. At column densities $N(\mathrm{H} \mathrm{I})$ exceeding $10^{17} \mathrm{~cm}^{-2}$, the gas becomes optically thick to ionizing radiation, and a discontinuity at the Lyman limit $(912 \AA)$ is detectable. In systems with $N(\mathrm{H} \mathrm{I})$ larger than $\sim 10^{19} \mathrm{~cm}^{-2}$, self-shielding renders the gas predominantly neutral. The damping wings of the Lorentzian component of the absorption profile begin to be detected from about the same column density, reaching their maximum in the "damped Ly $\alpha$ systems."

The question of whether the majority of the absorption systems are truly intervening at cosmological distances from the quasar, or ejected by it, which had received considerable interest in the earlier days, is now settled in favor of the intervening hypothesis. The huge momentum requirements for ejection (Goldreich \& Sargent 1976), the outcome of the Bahcall \& Peebles test (Bahcall $\&$ Peebles 1969, Young et al 1982a) for a random redshift distribution of absorbers to different QSOs, the discovery of galaxies at the same redshifts as metal absorption systems (Bergeron 1986), and the detection of high metallicity gas in systems close to the QSO and low metallicities more than $30,000 \mathrm{~km}^{-1}$ away from it (Petitjean et al 1994) leave no doubt that most of the systems are not physically related to the QSO against which they are observed.

The basic observational properties of the Ly $\alpha$ forest were established in the late 1970s and early 1980s, when the combination of 4-m telescopes (e.g. the AAT, KPNO, MMT, Palomar) and sensitive photon counting electronic detectors (e.g. the University College London's IPCS) first permitted quantitative spectroscopy on high redshift QSOs to be performed. Making use of the new technology, the work by Sargent et al (1980) set the stage for what for many years has been the standard picture of the Ly $\alpha$ forest: Ly $\alpha$ absorbers were found to be consistent with a new class of astronomical objects, intergalactic gas clouds, which are distinct from galaxies (and metal absorption systems) by their large rate of incidence $(\mathrm{d} \mathcal{N} / \mathrm{dz})$ and their weak clustering. Upper limits on the gas temperature and estimates for the ambient UV flux and for the cloud sizes were found to be consistent with a highly ionized $\left(n_{\mathrm{HI}} / n_{\mathrm{H}} \leq 10^{-4}\right)$ optically thin gas kept at a temperature $T \sim 3 \times 10^{4} \mathrm{~K}$ by photoionization heating. Sargent et al (1980) suggested that denser clouds in pressure equilibrium with a hotter (i.e. more tenuous) confining intercloud medium (ICM) could explain the apparent lack of change of these objects with time. It was argued that this picture matches the inferred cloud properties better than clouds held together by gravity, and there were a number of other appealing features. In the wake of the dark matter-based structure formation scenarios, the pressureconfined clouds have given way to models where Ly $\alpha$ clouds arise as a natural immediate consequence of gravitational collapse. These results are discussed later. 
In an earlier review, Weymann et al (1981) introduced a classification of absorption systems that is still useful, although some of the distinction has been blurred by the most recent research (Cowie et al 1995, Tytler et al 1995). In particular, the earlier review distinguished two classes of absorption systems, physically separated from the QSO environment, according to whether they do, or do not, show metal absorption lines in addition to the ubiquituous $\operatorname{Ly} \alpha$. For most of the $\operatorname{Ly} \alpha$, clouds detectable with current technology [i.e. $N(\mathrm{H} \mathrm{I}) \gtrsim 10^{12} \mathrm{~cm}^{-2}$ ], metal lines with metallicities common at high redshifts $\left(Z \lesssim 10^{-2} Z_{\odot}\right)$ are simply below the detection threshold. Therefore, this classification is simply an observational one. Rather than explore the nature of the division, if appropriate, between metal absorbers and Ly $\alpha$ systems, here we concentrate on the low column density absorbers. The study of metal absorption systems, possibly of great relevance to galaxy formation, is left for future review.

Below, we first discuss observational techniques and observed properties of Ly $\alpha$ systems (using the terms absorption systems, absorbers, and clouds interchangeably). Then we turn to various models of $\operatorname{Ly} \alpha$ absorbers, and finally we address some recent developments, and speculate about future developments.

\section{OBSERVATIONAL APPROACHES}

\subsection{Technical Possibilities, Observational Constraints}

Observational progress with traditional Ly $\alpha$ studies can conveniently be charted in terms of two limiting factors: the spectral resolution and the signal-to-noise ratio. The early (photographic) spectroscopy in the 1960s typically had to rely on resolutions of $10-20 \AA$. At optical wavelengths, this is barely sufficient to resolve velocity dispersions characteristic of galaxy clusters. Later, in particular the combination of echelle spectrographs and charge-coupled device (CCD) detectors permitted QSO spectroscopy to be done with 4-m telescopes at resolutions as high as $R \sim 5 \times 10^{4}$. QSO observers have been quick to make use of these advances, as is obvious from the semantic drift of "high resolution": In 1979, it meant $0.8 \AA$ (Sargent et al 1979); in 1984, $0.25 \AA$ (Carswell et al 1984); and in 1990, $0.08 \AA$ (Pettini et al 1990). Naturally, the average signal-to-noise ratio per resolution element did not benefit from the increase in spectral dispersion, and a single high resolution QSO spectrum required an embarassingly large number of nights on a 4-m telescope. Thus various ways of extracting information from the $\mathrm{Ly} \alpha$ forest have been developed in parallel. Some were tailored to detailed analysis of individual, expensive, high resolution spectra (line profile fitting), while others could be applied more automatically to larger samples of low resolution data (mean absorption; equivalent width measurements). The 10-m Keck telescope with its powerful High Resolution Spectrograph (HIRES; Vogt et al 1994) brought signal-to-noise ratios of 
$\sim 100$ within reach of high resolution [full width at half maximum (FWHM) $\sim 7 \mathrm{~km} \mathrm{~s}^{-1}$ ] absorption line studies, rendering some of the low resolution approaches obsolete. The new limiting factor for large telescope spectroscopy is not resolution nor collecting area but human power-coping (intelligently) with the continuous stream of large data sets already, or soon to be, available from the Keck telescopes, the Magellan, the Hobby-Eberly, the ESO-VLT, the MMT, etc. Recently, progress has also come from extending the wavelength regime into the UV band with the Hubble Space Telescope (HST) and the Hopkins Ultraviolet Telescope (HUT). In particular, the advent of the HST with its high resolution UV spectrographs has helped to compensate to some extent for the fact that optical Ly $\alpha$ spectroscopy can only sample the universe at redshifts larger than $z \sim 2.5$. We can now study the absorber properties and the absorber-galaxy connection in the local universe and, at high redshift, the far-UV (rest frame) helium Ly $\alpha$ forest.

\subsection{Low Resolution Spectroscopy: Mean Absorption}

The most basic observable of the Ly $\alpha$ forest is the quantity originally sought by Gunn \& Peterson (1965), the flux decrement $D$, or the mean fraction of the QSO continuum absorbed. It has become standard practice to measure the mean flux decrement $D_{A}$ between the Ly $\alpha$ and $\operatorname{Ly} \beta$ emission lines (Oke \& Korycansky 1982):

$$
D_{A}=\left\langle 1-\frac{f_{\text {obs }}}{f_{\text {cont }}}\right\rangle=\left\langle 1-e^{-\tau}\right\rangle=1-e^{-\tau_{\text {eff }}},
$$

where $f_{\text {obs }}$ is the observed (= residual) flux, $f_{\text {cont }}$ the estimated flux of the unabsorbed continuum, and $\tau$ the resonance line optical depth as a function of wavelength or redshift. The absorption is measured against a continuum level usually taken to be a power law in wavelength extrapolated from the region redward of the Ly $\alpha$ emission line. A knowledge of the mean absorption allows us, for example, to determine the resulting broadband color gradients in a QSO or high redshift galaxy spectrum, as caused by the Ly $\alpha$ forest. Measurements of $D_{A}$ from large data sets were performed by (among others) Oke \& Korycansky (1982), Bechtold et al (1984), O’Brian et al (1988), Steidel \& Sargent (1987b), Giallongo \& Cristiani (1990), Dobrzycki \& Bechtold (1991), and Schneider et al (1991 and references therein). Obviously, at the price of getting only one number out of each QSO spectrum, this technique gives the most model-independent measurement possible.

With $D_{A}$ measurements available over a range of redshifts, the redshift evolution of the Ly $\alpha$ forest can be investigated. Here the concept of an effective optical depth $\tau_{\text {eff }}(z)$, as defined in Equation 1 becomes useful. If we characterize a $\operatorname{Ly} \alpha$ forest as a random distribution of absorption systems in column 
density $N$, Doppler parameter $b$, and redshift $z$ space, such that the number of lines per interval $d N, d b$, and $d z$ is given by $\mathcal{F}(N, b, z) d N d b d z$, then

$$
\tau_{\text {eff }}=\int_{z_{1}}^{z_{2}} \int_{b_{1}}^{b_{2}} \int_{N_{1}}^{N_{2}}\left(1-e^{-\tau(N, b)}\right) \mathcal{F}(N, b, z) d N d b d z
$$

(Paresce et al 1980), for a population of absorbers without spatial or velocity correlations. Assuming that the $N$ and $b$ distribution functions are independent of redshift, and the redshift evolution of the number density of lines can be approximated by a power law, we can write $\mathcal{F}(N, b, z)=(1+z)^{\gamma} F(N, b)$, and

$$
\tau_{\mathrm{eff}}(z)=\frac{(1+z)^{\gamma+1}}{\lambda_{0}} \int_{b_{1}}^{b_{2}} \int_{N_{1}}^{N_{2}} F(N, b) W(N, b) d N d b,
$$

where the rest frame equivalent width is given by $W=(1+z)^{-1} \lambda_{0}^{-1}$ $\int d \lambda\left(1-e^{-\tau}\right)$.

This relation enables us to measure the redshift evolution of the number density of Ly $\alpha$ forest clouds, $d \mathcal{N} / d z \propto(1+z)^{\gamma}$, from the redshift dependence of the effective optical depth $\left[\tau_{\text {eff }} \propto(1+z)^{\gamma+1}\right]$ even if we do not resolve the individual absorption lines (Young et al 1982b, Jenkins \& Ostriker 1991, Press et al 1993, Zuo 1993, Lu \& Zuo 1994). The results of this approach are discussed below in the section on Ly $\alpha$ forest evolution, together with the conclusions from line counting methods.

The largest uncertainties in $D$ or $\tau_{\text {eff }}$ are probably caused by our ignorance about the precise QSO continuum level, against which the absorption is measured. Additional errors stem from the amount of absorption contributed by metal lines, which often cannot be identified as such and then removed from low resolution Ly $\alpha$ spectra.

\subsection{Intermediate Resolution Spectroscopy: Line Counting}

At higher resolution, where it becomes possible to distinguish between discrete absorption lines, the distribution of the lines in terms of equivalent width $W$ and redshift $z$ is the next most sophisticated tool. Just as the mean absorption $D$ under certain conditions provides a measure of the mean (gas) density of the universe, so does the number of lines per unit equivalent width, $d^{2} \mathcal{N} / d W d z$, essentially measure the clumpiness of the Ly $\alpha$ forest gas. An exponential distribution in $W$ and a power law dependence on $(1+z)$ have been found to provide a reasonable match to the observed line counts (Sargent et al 1980, Young et al 1982b, Murdoch et al 1986). For lines above a rest equivalent width threshold $W>0.16 \AA$,

$$
\frac{d^{2} \mathcal{N}}{d W d z}=\frac{W}{W_{*}} e^{-W / W_{*}}(1+z)^{\gamma}
$$


with a typical $W_{*} \approx 0.27 \AA$ (Bechtold 1994), and $1.5<\gamma<3$ (see also the discussion on redshift evolution below). The multiplicative form of Equation 4 is justified by a relatively weak dependence of $W_{*}$ on $z$ (Murdoch et al 1986). The exponential model fits less well for lines with $W<0.3 \AA$, as the weaker lines are moving off the saturated part of the curve of growth and become more numerous.

Unfortunately, statistics involving the observed equivalent width distribution are not easy to interpret in physical terms. The $W$ values are usually obtained by simply measuring statistically significant downward excursions from the QSO continuum. Without properly deblending the lines (which is impossible at low resolution), the curve of growth cannot be used to relate $W$ to the more meaningful parameters $N$ and $b$.

\subsection{High Resolution Spectroscopy: Voigt Profile Decomposition}

If the Ly $\alpha$ forest is seen as an assemblage of redshifted lines, the standard arsenal of notions and techniques from stellar spectroscopy becomes applicable. For lower resolution data, the equivalent width provides a combined measure of line width and strength. In high resolution spectra (FWHM $<25 \mathrm{~km} \mathrm{~s}^{-1}$ ) where the typical Ly $\alpha$ line is resolved, the line shapes are found to be reasonably well approximated by Voigt profiles (Carswell et al 1984). Then line width [Doppler parameter $b\left(=2^{1 / 2} \sigma\right)$ ], column density $N(\mathrm{H} \mathrm{I})$, and redshift $z$ of an absorption line are the basic observables. The statistics of the Ly $\alpha$ forest from high resolution studies largely have been cast in terms of the distribution functions of these three quantities and their correlations. The main advantage of the high resolution approach is the opportunity of determining the shape of these distribution functions without parametric prejudices, by directly counting lines with parameters in a certain range.

The standard approach to Voigt profile fitting (Webb 1987, Carswell et al 1987) relies on $\chi^{2}$ minimization to achieve a complete decomposition of the spectrum into as many independent Voigt profile components as necessary to make the $\chi^{2}$ probability consistent with random fluctuations. For stronger Ly $\alpha$ lines, the higher order Lyman lines can provide additional constraints when fitted simultaneously. The absorption lines are measured against a QSO continuum estimated locally from polynomial fits to spectral regions deemed free of absorption. A local high order continuum fit (as compared to a global extrapolation with a physical model for the QSO continuum) is necessary because the spectra are patched together from many individual echelle orders with strong variations in sensitivity. These variations do not divide out completely when dividing by the flux of a standard star because the light going through a slit narrow enough to ensure slit-width-limited resolution varies with the seeing 
conditions and with the position of the object on the slit. When applying a local fit to the continuum, the zero order contribution tends to be underestimated, i.e. the continuum is drawn too low, which is the main drawback of this method.

Given sufficient spectral resolution, and assuming that Ly $\alpha$ clouds are discrete entities (in the sense of some of the models discussed below), the profile fitting approach is the most physically meaningful way of extracting information from the $\mathrm{Ly} \alpha$ forest. If the absorber is a gas cloud with a purely Gaussian velocity dispersion (a thermal Maxwell-Boltzmann distribution, plus any Gaussian contributions from turbulence), a Voigt profile provides an exact description of the absorption line shape. The Doppler parameter can then be written as the quadratic sum of its individual contributions:

$$
b=\sqrt{\frac{2 k T}{m}+b_{\text {turb }}^{2}} \text {. }
$$

Unfortunately, in most more realistic models of the absorbing gas, finite velocity and density gradients invalidate the assumptions underlying the Voigt profile fitting, and the line parameters may have less immediate physical meaning. Departures of the absorption line shape from a Voigt profile may contain valuable information about the underlying nature of the absorption systems, and different scenarios may have quite different observational signatures. Rotational motion (Weisheit 1978, Prochaska \& Wolfe 1997), gravitational collapse (McGill 1990, Meiksin 1994, Rauch 1996), and galactic outflows (Fransson \& Epstein 1982, Wang 1995) have been discussed in terms of the likely absorption line shapes they produce. As yet, the quantitative application of these results has proven difficult because of the lack of realistic prototypical models for the actual line formation, the rather subtle departures from Voigt profiles expected, and the wide variety of profiles actually encountered.

Non-Voigt profiles can still be fitted as blends of several Voigt profiles, but the information about the nonthermal motion is encoded in the spatial correlations between the individual profiles (Rauch 1996). Also, there is no guarantee that the number of components necessary for a good fit converges with increasing signalto-noise ratio. Clearly, for specific line formation models, the results of Voigt profile decomposition may have less immediate physical meaning, and global techniques of extracting the velocity information may be more appropriate.

\section{OBSERVATIONAL RESULTS}

\subsection{Redshift Evolution of the Lyman Alpha Forest}

In an individual QSO LOS, observations of the high redshift $(z \sim 3)$ Ly $\alpha$ forest can extend over a redshift range $\Delta z$ greater than unity. Then we are sampling a significant fraction of a Hubble time, and it is natural to expect to see changes 
in the absorption pattern, e.g. in the rate of incidence of absorption lines with redshift or in the mean optical depth.

EVOLUTION OF THE LINE DENSITY An analytic expression (Wagoner 1967, Bahcall \& Peebles 1969) can be given for the number of absorption systems per unit redshift, $d \mathcal{N} / d z$, in terms of the comoving number density $n_{0}(z)$ of absorbers, the geometric absorption cross section $\sigma(z)$, and the Hubble constant $H_{0}$ :

$$
\frac{d \mathcal{N}}{d z}=\frac{c n_{0}(z) \sigma(z)}{H_{0}} \frac{1+z}{\left(1+q_{o} z\right)^{1 / 2}} .
$$

For absorbers with no intrinsic evolution,

$$
\frac{d \mathcal{N}}{d z} \propto\left\{\begin{array}{ll}
1+z, & q_{0}=0 \\
(1+z)^{1 / 2}, & q_{0}=\frac{1}{2}
\end{array} .\right.
$$

The transformation from redshift $z$ to the coordinate $X(z)=\int_{0}^{z}(1+z)^{-1}(1+$ $\left.2 q_{0} z\right)^{1 / 2} d z$ (Wagoner 1967) is sometimes used to take out the mere cosmological redshift dependence, such that $d \mathcal{N} / d X=c n_{0}(z) \sigma(z) H_{0}^{-1}$.

Peterson (1978) first pointed out an increase in the number of Ly $\alpha$ clouds with redshift beyond what was expected for a population of nonevolving objects. At first this result was subject to some debate (see the summary by Murdoch et al 1986), but it is now clear that the Ly $\alpha$ forest as a whole evolves quite strongly with $z$. The observationally determined evolution in the number of absorbers above a certain column density threshold is usually expressed in the form

$$
\frac{d \mathcal{N}}{d z}=\left(\frac{d \mathcal{N}}{d z}\right)_{0}(1+z)^{\gamma},
$$

(Sargent et al 1980, Young et al 1982b), where the exponent includes the cosmological dependence. Much observational effort has been devoted to studying the redshift number density evolution, but unfortunately, the resulting conclusions are far from robust. This is because Ly $\alpha$ cloud column densities $N$ are distributed according to a power law $N^{-\beta}$ with index $\beta \sim 1.5$ (see below), so the majority of lines in any column density-limited sample are always close to the threshold, and small variations in imposing the threshold can cause large changes in the estimated numbers of lines and in $\gamma$. Moreover, line blending and its dependence on spectral resolution, data quality, and redshift can make a large difference in the normalization $(d \mathcal{N} / d z)_{0}$, with individual studies differing by a factor of two or more (the interested reader may refer to Liu \& Jones 1988, Parnell \& Carswell 1988, Trevese et al 1992, and Kim et al 1997 for a discussion of blending effects). Every individual study has a finite redshift range available so that the uncertainty in the normalization is correlated 
with the exponent $\gamma$; the differences between the observed values of $\gamma$ exhibit a disturbingly large scatter. Thus the line counting approach is unsatisfactory when it comes to redshift evolution. Rather than discussing the many individual (and sometimes mutually inconsistent) contributions made to this question, we outline some typical results as follows.

At lower resolution (FWHM $\sim 50-100 \mathrm{~km} \mathrm{~s}^{-1}$ ), large samples of lines have been used to investigate this topic. The $\gamma$ values tend to lie between the low value, $\gamma=1.89 \pm 0.28$, obtained by Bechtold (1994) $(W>0.32 \AA)$ and the high one, $\gamma=2.75 \pm 0.29$ (for $W>0.36 \AA$ ), from the study by Lu et al (1991). High resolution spectra, usually confined to $z>2$, tend to give an equally wide range of values: $\gamma=1.7 \pm 1.0(W>0.2 \AA$ Atwood et al 1985); $2.9 \pm 0.3$ $(2<z<4.5$; Cooke et al 1997); $\gamma=2.78 \pm 0.71(\log N>13.77 ; 2<z<3.5$; Kim et al 1997). At $z>4$, the evolution appears to be accelerating, with $\gamma$ increasing from a value just below 3 to 5.5 (Williger et al 1994).

EVOLUTION OF THE MEAN ABSORPTION Similar numbers are obtained by methods that do not depend on line counting. For $2.5<z<4.5$, Press et al (1993), measuring $\tau_{\text {eff }}(z)$, derived $\gamma=2.46 \pm 0.37$. Zuo \& Lu (1993), deriving $D_{A}$ from spectra reconstituted from published absorption line lists, found $\gamma=2.87 \pm 0.23$, but they considered a broken power law with $\gamma=2.82$ below and 5.07 above $z=3.11$ to give a better fit, which is in agreement with an upturn at the highest redshifts. The evolution of the mean absorption $\tau_{\text {eff }}$ or $D_{A}$ is indeed a more robust measure of change, but its relation to the number of clouds is not entirely straightforward, as a model must be relied on for the distribution of absorbers, $\mathcal{F}(N, b, z)$, the functional form of which is a priori unknown. Although usually not taken into account, there clearly is mutual dependence of $N$, $b$, and $z$ in the form of clustering and of differential evolution in $N$ and $b$. Moreover, the column density of the lines dominating the absorption changes with redshift [at $z \sim 3$, lines with $\log N(\mathrm{H} \mathrm{I}) \sim 14$ contribute most], and so does the range of column densities to which the measurements of $\tau_{\text {eff }}$ are most sensitive.

To the surprise of many, the first studies with HST of low redshift $(z<1.5)$ Ly $\alpha$ lines (Bahcall et al 1991, 1993, Morris et al 1991, Impey et al 1996) (see also the paragraph on the low $z$ Ly $\alpha$ forest below) have discovered more absorption systems than expected from a naive extrapolation of any of the high $z$ power law exponents. Moreover, the low $\gamma=0.48 \pm 0.62$ valid from $z \sim 0$ to $z \sim 1$ gives a $d \mathcal{N} / d z$ that is consistent with a constant comoving density of objects. Accordingly, single power law fits attempting to explain both high and low $z$ absorbers fail to account for the rapid upturn around $z \sim 1$ (e.g. Impey et al 1996).

DIFFERENTIAL EVOLUTION AS A FUNCTION OF LINE STRENGTH A number of authors have made the case for differential evolution, as a function of column 
density or equivalent width. At the high column density end, Lyman limit systems $(\log N>17)$ were found to be consistent (Lanzetta 1988, Sargent et al 1989 ) with a nonevolving population at least out to $z=2.5$. Therefore, the lower column density systems must be evolving faster, given the above $\gamma$ values. Murdoch et al (1986) and Giallongo (1991) noted a general tendency toward slower evolution of $\gamma$ with increasing $W$ threshold, which could provide continuity between the large $\gamma$ for the general line population and the nonevolving, optically thick Lyman limit regime. However, other studies claim the opposite trend. Bechtold (1994) found a $\gamma$ that increased from $1.32 \pm 0.24$ for weaker lines $(W>0.16 \AA)$ to $1.89 \pm 0.28$ for strong lines $(W>0.32 \AA)$. Similar conclusions were reached by Acharya \& Khare (1993). Bechtold's weakest lines $(0.16<W<0.32 \AA)$ are consistent with no evolution at all, a result that is in agreement with the Keck study by Kim et al (1997). The latter group and Giallongo (1991) based their conclusions on high resolution data, whereas the other papers cited are based on low resolution. Blending cannot be the whole explanation, since the present discrepancy persists regardless of resolution.

CONCLUSIONS We may summarize the more secure results on number evolution as follows: Going from $z=0$ to $z \sim 1$, there is no obvious change in the comoving number of the clouds. Then between $1<z<2$ a steep rise sets in, which can be reasonably described by a power law $(1+z)^{\gamma}$ with index $2<\gamma<3$. At redshifts approaching $z \sim 4$, the upturn appears to steepen further. Thus a single power law does not fit the curvature of the $d \mathcal{N} / d z$ versus $z$ relation well. Differential evolution, with stronger lines evolving less rapidly, must exist to reconcile the large $\gamma$ for most of the forest lines with the nonevolving population of Lyman limit absorbers. Some studies suggest that the line density at the very low column density range does not evolve much either, in which case the rapid evolution in between is caused either by a genuine, rapidly changing subpopulation or by biases in the analysis that we do not understand properly. In any case, the average optical depth is evolving strongly with $\tau_{\text {eff }} \propto(1+z)^{\gamma+1}$ and $\gamma$ around 2-3 (for $z \gtrsim 3$ ).

\subsection{The Proximity Effect: Measuring the Ionizing Ultraviolet Background}

The UV radiation from QSOs has been considered as the most natural origin for the ionization of the intergalactic gas (Arons \& McCray 1969, Rees \& Setti 1970). The finite number density of QSOs suggests that there may be inhomogeneities in the ionization state of the Ly $\alpha$ clouds near each QSO. The term proximity effect (coined by Bajtlik et al 1988) refers to a relative lack of Ly $\alpha$ absorption in the vicinity of the background QSO. The effect was first discussed by Weymann et al (1981), who also suggested the currently accepted explanation of increased ionization of the clouds by the nearby QSO. Carswell 
et al (1982) realized that the general increase of the absorption line density $d \mathcal{N} / d z$ with redshift was accompanied by a simultaneous decrease of $d \mathcal{N} / d z$ in each individual QSO spectrum when approaching the QSO's emission redshift. Murdoch et al (1986), calling it the inverse effect, confirmed and clarified this result. Tytler (1987b), referring to the effect as the anomaly, questioned the specific assumption that the reduced absorption is caused by the QSO's radiation field. Hunstead et al (1988) and Webb \& Larsen (1988) defended the earlier interpretation and, in particular, the claim that the effect is local to the neigborhood of the QSO.

If the proximity effect is indeed caused by enhanced ionization, measurement of the intensity of the ionizing UV background becomes possible from observations of the density of lines, $d \mathcal{N} / d z$, as a function of the distance from the QSO. Let us assume that in the vicinity of a QSO, $d \mathcal{N} / d z$ is reduced, presumably owing to the excess ionization of the gas. With increasing distance from the emission redshift, the QSO's ionizing flux decreases until the UV background intensity begins to dominate the ionization of the intergalactic gas. For example, at the point where the background intensity equals the QSO flux, $L_{Q}\left(4 \pi r_{L}^{2}\right)^{-1}$ (known from photometry), the neutral column density of a cloud should be lower by a factor of one half, with a corresponding decrease in $d \mathcal{N} / d z$ for lines above a given detection threshold. In this way, Carswell et al (1987) performed the first crude measurement of the UV background radiation field, obtaining $J_{-21}=3$, where $J=J_{-21} \times 10^{-21} \mathrm{erg} \mathrm{cm}^{-2} \mathrm{~s}^{-1} \mathrm{~Hz}^{-1} \mathrm{sr}^{-1}$ is the intensity at the Lyman limit, $912 \AA$. Bajtlik et al (1988) confirmed this result from a larger low resolution sample of 19 QSOs, obtaining $J_{-21}=1_{-0.7}^{+3.2}$. Their measurement procedure (adopted by most later studies) consists of fitting the number density of lines per unit redshift distance $X_{\gamma}=\int(1+z)^{\gamma} d z$

$$
\frac{d \mathcal{N}}{d X_{\gamma}}=\left(\frac{d \mathcal{N}}{d z}\right)_{0}\left(1+\frac{L_{Q}}{16 \pi^{2} r_{L}^{2} J}\right)^{1-\beta}
$$

as a function of the luminosity distance $r_{L}$, where the background intensity $J$ is the quantity desired. The quantity $\beta$ is again the exponent of the power law distribution of column densities. Lu et al (1991) get identical results with a larger sample. Bechtold (1994), in her even larger data set, found $J_{-21}=3$. The largest compilations of high resolution data gave $J_{-21}=0.5 \pm 0.1$ (Giallongo et al 1996) and $J_{-21}=1_{-0.3}^{+0.5}$ (Cooke et al 1997). None of these studies has found evidence for a significant change with redshift (for $1.6<z<4.1$ ). However, Williger et al (1994) and Lu et al (1996) both found lower values $\left(J_{-21}=0.2\right)$ just above $z \sim 4$, in contrast to Savaglio et al's (1997) value $\left(J_{-21}=0.7\right)$ for the same redshift, which is consistent with no evolution. In any case, when going to lower redshifts, there appears to be a drastic decline in intensity below $z \sim 1$.6. At $\langle z\rangle \sim 0.5$, Kulkarni \& Fall (1993) obtained $J_{-21}=6 \times 10^{-3}$ from HST FOS data (Bahcall et al 1993). 
SYSTEMATIC EFFECTS? Assuming that the proximity effect really does measure the background intensity and not some other environmental effect caused by the QSO (e.g. suppression of neutral gas absorption in a high pressure environment), we still know this quantity only to within an order of magnitude, and the uncertainty may be even larger than that. This is because, in addition to the errors from line counting discussed in the previous section, there are systematic uncertainties in the QSO flux.

Espey (1993) has quantified the overestimate in the local QSO flux, which arises from the well-known systematic difference between the "actual" redshift of the QSO (as determined from narrow forbidden lines) and the redshift of the (usually blueshifted) broad emission lines that define the "QSO redshift" used normally for the proximity effect. The blueshift typically may amount to $\sim 1500 \mathrm{~km} \mathrm{~s}^{-1}$. Downward corrections for $J$ correspond to a factor of 2-3 in this case. These corrections have been taken into account in the work by Williger et al (1994), Lu et al (1996), and Cooke et al (1997), but they would reduce, for example, Bechtold's value by a factor three.

Other uncertainties that are more difficult to quantify (see the review by Bechtold 1995) include QSO variability on the ionization time scale of the gas (Bajtlik et al 1988), gravitational lensing (amplification of the QSO luminosity), and uncertainties in the shape of the column density distribution (Chernomordik \& Ozernoy 1993). Gravitational clustering of clouds near the QSOs may cause an excess number of clouds, which again would lead to an overestimate of the background intensity by a factor of up to three (Loeb \& Eisenstein 1995).

The proximity effect measurements beg the question whether there are enough QSOs to produce the ionizing background seen or whether an additional population of sources is needed. We may conclude as did Espey (see Espey 1993, and references therein) that the contribution of known QSOs to the background intensity probably agrees within the errors with the intensity from this measurement.

A related debate on whether the QSOs can ionize the IGM has occupied an even larger space in the literature. Unfortunately, we cannot do justice to this extended discussion here but cite some of the references in the section on the He II Ly $\alpha$ forest below.

\subsection{Absorption Line Widths}

By measuring the line widths, we may gain insights into the temperature and kinematics of Ly $\alpha$ clouds. High resolution spectra showed that many low column density $\left(N_{\mathrm{H} \mathrm{I}}<10^{15} \mathrm{~cm}^{-2}\right)$ Ly $\alpha$ lines do indeed show widths $\left[b\left(=2^{1 / 2} \sigma\right)\right.$ $\sim 10-45 \mathrm{~km} \mathrm{~s}^{-1}$ ] that are consistent with photoionization temperatures (Chaffee et al 1983, Carswell et al 1984; see review by Carswell 1988), though some lines appear to be as wide as $100 \mathrm{~km} \mathrm{~s}^{-1}$. Median Doppler parameters are around $b_{\text {med }} \sim 30-35 \mathrm{~km} \mathrm{~s}^{-1}$, with a largely intrinsic scatter about the mean 
with standard deviation $\sim 15 \mathrm{~km} \mathrm{~s}^{-1}$ (Atwood et al 1985, Carswell et al 1991, Rauch et al 1992). The Doppler parameters may decrease with increasing redshift. Williger et al (1994) have found an excess of lines with lower Doppler parameters $b \sim 20 \mathrm{~km} \mathrm{~s}^{-1}$ at $z \approx 4$.

Occasionally, a correlation between Doppler parameter and column density has been noted (Carswell et al 1984, Hunstead et al 1988). However, the reality of this effect was subject to a debate that culminated in the so-called $b-N$ controversy (Hunstead \& Pettini 1991, Peacock 1991, Webb \& Carswell 1991), when Pettini et al (1990) suggested that, when looked at with high enough resolution, Ly $\alpha$ lines had much lower Doppler parameters (mostly $b \lesssim 22 \mathrm{~km} \mathrm{~s}^{-1}$ ) than previously thought. There also appeared to be a strong positive correlation between Doppler parameter $b$ and column density $N$. These results were not confirmed by an analysis of another QSO spectrum with an identical observational setup (Carswell et al 1991). The controversy was resolved when it was realized that the presence of noise in a spectrum can distort weak line profiles and lead to underestimates of the average $b$ values of low column density lines. The problem is exacerbated by a detection bias against weak broad lines, which are more difficult to find against a noisy continuum and tend to end up below the detection threshold. The combination of these effects accounts for both the presence of spuriously low Doppler parameters and the apparent $b-N$ correlation seen in these data sets (Rauch et al 1992, 1993).

RECENT KECK RESULTS Data taken at similar resolution but with much higher signal-to-noise ratio with the Keck telescope's HIRES instrument basically have confirmed the earlier 4-m results. Hu et al (1995) found the Doppler parameter distribution at $z \sim 3$ to be well represented by a Gaussian with a mean of $28 \mathrm{~km} \mathrm{~s}^{-1}$ and width of $\sigma=10 \mathrm{~km} \mathrm{~s}^{-1}$, truncated below a cutoff $b_{c}=20 \mathrm{~km} \mathrm{~s}^{-1}$. With increasing redshift, there seems to be a genuine trend to lower Doppler parameters. The finding by Williger et al (1994) of evolution in $b$ appears confirmed: Median Doppler parameters for relatively strong lines $(13.8<\log N(\mathrm{H} \mathrm{I})<16.0)$ change from $41 \mathrm{~km} \mathrm{~s}^{-1}(\langle z\rangle \sim 2.3$; Kim et al 1997) to $31 \mathrm{~km} \mathrm{~s}^{-1}$ ( $\langle z\rangle \sim 3.7$; Lu et al 1996), with lower cutoffs dropping from 24 to $15 \mathrm{~km} \mathrm{~s}^{-1}$ over the same redshift range. The locus of the Pettini et al (1990) narrow lines in the $b-N$ diagram is virtually empty (Hu et al 1995), as expected in data with such a high signal-to-noise ratio. Kirkman \& Tytler (1997) obtained similar results for the Doppler parameters in their Keck data at even better signal-to-noise ratios, but they question the significance of the change with redshift, and find a lower mean $b$ of $23 \mathrm{~km} \mathrm{~s}^{-1}(\langle z\rangle \sim 2.7)$, with a lower cutoff $b_{c}=14 \mathrm{~km} \mathrm{~s}^{-1}$ at $\log N(\mathrm{H} \mathrm{I})=12.5$. However, at $\log N(\mathrm{H} \mathrm{I})=13.8$, their minimum $b$ at $19 \mathrm{~km} \mathrm{~s}^{-1}$ is very close to the result of Kim et al (1997), so the analyses may well be consistent. It is conceivable that the discrepancies at lower 
column densities arise once more from the noise bias discussed above, which may affect any data set as long as there continues to be a supply of weaker and weaker lines crossing the detection threshold with increasing signal-to-noise ratio. The differences might lie in a different understanding of what constitutes "statistically acceptable fits" or "detectable lines." A data set spanning a large redshift range, with—most importantly—a homogeneous signal-to-noise ratio would be desirable.

THE TEMPERATURE OF THE INTERGALACTIC MEDIUM FROM LINE PROFILES? Though narrow lines $\left(b<15 \mathrm{~km} \mathrm{~s}^{-1}\right)$ are apparently very rare or even absent, this should not be interpreted as indicating a minimum temperature of the Ly $\alpha$ absorbing gas. The issue is more complex; in analogy with other astrophysical situations, there are reasons for which a correlation might be expected between the temperature (or velocity dispersion) and the density (or column density) of the gas. Typical photoionization equilibrium temperatures should be in excess of 30,000 K (e.g. Donahue \& Shull 1991), but temperatures as low as 20,000 K can be attained through inverse Compton cooling and a decrease of the ionizing spectrum at the He II edge (Giallongo \& Petitjean 1994). If photothermal equilibrium is abandoned, adiabatic expansion cooling can lower the temperatures further while maintaining high ionization, as suggested by Duncan et al (1991). Currently favored theories of Ly $\alpha$ clouds that are the result of cold dark matter (CDM)-based gravitational collapse do predict a $b-N$ correlation with temperatures for low column density clouds even below $10^{4} \mathrm{~K}$, as a consequence of adiabatic expansion and inefficient photoheating at low densities, while the larger column densities are heated as a result of compression during collapse. However, in spite of the low temperatures, the Doppler parameters of the weak lines are predicted to be large because of bulk motion: Nature, in a random act of unkindness, has endowed these cool clouds with a large size so that the Hubble expansion dominates the line broadening. Yet it may be worth trying to track down the residual influence of the gas temperature on the line profiles. The lower column density systems are at gas densities where the cooling time (for processes other than expansion cooling) exceeds a Hubble time. Therefore, the gas retains a memory of the temperature after reheating is complete (Meiksin 1994, Miralda-Escudé \& Rees 1994, Hui \& Gnedin 1997), and the process of reheating may have left a record in the Doppler parameter distribution (Haehnelt \& Steinmetz 1997).

\subsection{The Column Density Distribution}

In absorption line studies, the column density distribution function (CDDF), i.e. the number of absorbers in a given column density bin, occupies a similarly central place (and provides similarly vague information) as the luminosity 
function in the study of galaxies. The observational determination of the CDDF relies on a patchwork of techniques, owing to the large dynamic range of observable $\mathrm{H}$ I absorption. The CDDF can be measured relatively unambiguously from Voigt profile fitting for column densities between $N(\mathrm{H} \mathrm{I})$ greater than a few times $10^{12} \mathrm{~cm}^{-2}$, the detection limit for typical Keck spectra, to about a few times $10^{14} \mathrm{~cm}^{-2}$, where the linear part of the curve of growth ends. The weakest lines $\left[N(\mathrm{H} \mathrm{I}) \lesssim 10^{13} \mathrm{~cm}^{-2}\right]$ are so numerous that they begin to overlap, requiring application of confusion corrections (Hu et al 1995). Above $\sim 10^{14} \mathrm{~cm}^{-2}$, once a line is saturated, there is a certain degeneracy between a small change in apparent line width and a large change in column density. Then the column densities are relatively difficult to measure exactly by any means. The situation is complicated further by the appearance of noticeable multicomponent structure in absorption systems with column densities above $10^{15} \mathrm{~cm}^{-2}$ (e.g. Cowie et al 1995). Blending among these components due to the large thermal width of the (already saturated) Ly $\alpha$ can mimick large Doppler parameter/high column density lines. Simultaneous fits to the higher order Lyman lines (which are less saturated owing to their lower oscillator strengths) can only help to some degree. Eventually, for systems with column density $N(\mathrm{H} \mathrm{I}) \gtrsim 10^{17} \mathrm{~cm}^{-2}$, the discontinuity at the Lyman limit (LL) can be observed $\left(10^{17.3} \mathrm{~cm}^{-2}\right.$ corresponds to $\tau_{L L} \sim 1$ ), again giving a relatively precise measure of the H I column (Lanzetta 1988, Sargent et al 1989). From $N \gtrsim 10^{18.5}$ on, the damping wings of Ly $\alpha$ become detectable. The line width is now entirely a measure of the column density and again can be read off by Voigt profile fitting or, in lower resolution data, directly from the equivalent width (Wolfe et al 1986).

Carswell et al (1984) found that the number of absorbers $\mathcal{N}$ per unit H I column density interval can be parametrized as

$$
\frac{d \mathcal{N}}{d N} \propto N^{-\beta}, \quad \beta=1.68, \quad \text { and } \quad 13<\log N<15 .
$$

Tytler (1987a) has drawn attention to the remarkable fact that when results from higher column density surveys are included, a single power law with slope $\beta=1.5$ fits the whole range of observable column densities well. Keck spectra (Hu et al 1995, Lu et al 1996, Kim et al 1997, Kirkman \& Tytler 1997) appear to show that the power law extends over 10 orders of magnitude in column density from $10^{12}$ to $10^{22} \mathrm{~cm}^{-2}$ (if the confusion correction made at the low column density end is justified). A customary working definition of the CDDF (Tytler 1987a) is

$$
f(N)=\frac{\mathcal{N}}{\Delta N \sum_{i} \Delta_{i} X},
$$

which gives the total number $\mathcal{N}$ of absorbers in $\mathrm{H}$ I column density bin $[N, N+$ $\Delta N$ ], found over the total surveyed redshift distance $\sum_{i} \Delta_{i} X$, where $X$ has been 
defined earlier. One of the more recent measurements (Hu et al 1995) gives

$$
f(N)=4.9 \times 10^{7} N^{-1.46}, \quad 12.3<\log N<14.5 .
$$

Various authors (Bechtold 1987, Carswell et al 1987, Giallongo et al 1993, Meiksin \& Madau 1993, Petitjean et al 1993) have presented evidence for departures from a single power law that seem to be borne out by the new Keck data. A steepening for $\log N \gtrsim 14$ explains why individual high resolution spectra tend to yield $\beta \sim 1.7-1.8$ for the regions $(\log N \sim 13-15)$ for which they are most sensitive (e.g. Carswell et al 1984, 1987, Atwood et al 1985, Rauch et al 1992). In the high column density range (beyond $\log N \sim 16$ ), $f(N)$ flattens and damped systems are more abundant than they should be, judging from an extrapolation of the lower column density power law. Weak evolution of $f(N)$ may occur in the sense that this turnover moves slightly with redshift (Carswell et al 1987, Kim et al 1997), but the evidence currently is not overwhelming, given that the dip in $f(N)$ occurs in a column density range, where the determination of $N(\mathrm{H} \mathrm{I})$ is least certain (see above).

\subsection{Spatial Structure Along the Line of Sight: Clustering and Voids}

Measurements of the two-point correlation function (TPCF) in velocity space along the LOS, $\xi(\Delta v)$, led Sargent et al (1980) to conclude that Ly $\alpha$ clouds are not clustered as strongly as galaxies. Given the probability $\Delta p$ of finding a pair of clouds with absorption cross section $\sigma$ and space density $n_{0}(z)$, separated by a velocity interval $\Delta v, \xi(\Delta v)$ is given by the following expression:

$$
\Delta p=n_{0} \sigma \Delta v[1+\xi(\Delta v)],
$$

where $\Delta v=c \Delta z /(1+\bar{z})$ is the velocity splitting in the rest frame at mean red$\operatorname{shift} \bar{z}$. No correlation signal was found on scales of $\Delta v$ between 300 and 30,000 $\mathrm{km} \mathrm{s}^{-1}$. Clustering for small line pair splittings $\left(\Delta v \lesssim 300 \mathrm{~km} \mathrm{~s}^{-1}\right)$ would still be consistent with this result, given the limited resolution and the effects of blending caused by the large line widths. Based on Voigt profile fits to high resolution data, Webb (1986) obtained the first evidence for the presence of a weak clustering signal $\left[\xi\left(100 \mathrm{~km} \mathrm{~s}^{-1}\right) \approx 0.5\right.$, at $\left.z \sim 2.5\right]$ at small separations. This result has been confirmed by others (e.g. Muecket \& Mueller 1987, Ostriker et al 1988). It is hard to detect the clustering at a high level of significance in any individual QSO spectrum because of the short redshift path length, and both detections and nondetections have been reported (Rauch et al 1992, Kulkarni et al 1996). A variety of techniques seem to indicate, however, that there really is weak clustering in the $z \sim 3$ forest on small scales. If Ly $\alpha$ lines are considered as blends of components with intrinsically narrower line widths, the clustering amplitude could be much higher (Rauch et al 1992). In particular, the strong clustering seen 
among metal absorption lines is largely invisible in Ly $\alpha$ because of blending between the saturated Ly $\alpha$ components associated with the metals. It is perhaps not that surprising that a Ly $\alpha$ clustering amplitude, increasing with $\mathrm{H}$ I column density, was actually reported (Chernomordik 1994, Cristiani et al 1995, 1997) and has been related to the clustering seen in metal absorption lines (Cowie et al 1995, Fernandez-Soto et al 1996). Earlier, Crotts (1989), by measuring the correlation in real space across the sky among systems in muliple LOS, had reported an increase of clustering with $\operatorname{Ly} \alpha$ equivalent width (at $z \sim 2.5$ ).

STRUCTURE ON VARIOUS SCALES The amplitude of the TPCF is not the only tool for measuring structure in the forest. In low resolution data, blends caused by clustering show up as a distortion in the equivalent width distribution of $\mathrm{Ly} \alpha$ lines, such that in the clustered case, there are more large equivalent width lines and fewer small ones than for a random distribution of lines in velocity space (Barcons \& Webb 1991). A number of other approaches, mostly equivalent to the hierarchy of correlation functions or parts thereof (White 1979), may give a more robust clustering signal on small scales by including higher order correlations. Especially, the void probability function and, more generally, neighbor statistics (Ostriker et al 1988, Liu \& Jones 1990, Fang 1991, Meiksin \& Bouchet 1995) have been used, invariably revealing the non-Poissonian (clustered) nature of the distribution of clouds in velocity space (Ostriker et al 1988, Bi et al 1989, Liu \& Jones 1990, Babul 1991).

Structure has been detected on many different scales, in addition to the smallscale clustering described above. Fang (1991) used a Kolmogorov-Smirnov test for nearest neighbor intervals to detect a signal on scales of $30-50 \mathrm{~h}^{-1} \mathrm{Mpc}(\mathrm{h}$ is the Hubble constant in units of $100 \mathrm{~km} \mathrm{~s}^{-1} \mathrm{Mpc}^{-1}$ ). Mo et al (1992), from an analysis of the extrema in the slope of the TPCF, saw structure at 60 and $130 \mathrm{~h}^{-1}$ Mpc. Meiksin \& Bouchet (1995) found an anticorrelation in the TPCF around 3-6 $\mathrm{h}^{-1}$ Mpc. Pando \& Fang (1996), applying the wavelet transform, found clusters $\sim 20 \mathrm{~h}^{-1} \mathrm{Mpc}$ in size in the Ly $\alpha$ forest. The physical interpretation of the various results is not entirely obvious. The usefulness of the data for large-scale structure analyses has always been accepted at face value, and it would certainly be entertaining to see whether there are systematic effects in the data, perhaps causing some of the structure. There are intrinsic scales in the spectra (like the quasiperiodic change in signal-to-noise ratio caused by the sensitivity maxima of the orders in an echelle spectrum) that are of similar magnitude $\left(\sim 5000 \mathrm{~km} \mathrm{~s}^{-1}\right.$, or $\sim 25 \mathrm{~h}^{-1} \mathrm{Mpc}$, comoving at $\left.z \sim 3\right)$ as some of the above detections.

Most of the clustering work is based on analyzing correlations between distinct absorption lines. Including information about the relative strength of the absorption as a function of velocity splitting can improve the significance of any correlations, and give clues to the mechanism causing the signal. Webb \& 
Barcons (1991) and Zuo (1992) have suggested correlating equivalent widths, rather than just lines above a detection threshold, to search for inhomogeneities in the gas pressure or ionizing flux along the LOS. Fardal \& Shull (1993), Press et al (1993), and Zuo \& Bond (1994) have extended this approach to statistical models of the pixel intensity correlations, a technique useful for disentangling line widths and small-scale clustering on overlapping scales.

VOIDS IN THE FOREST A specific discussion revolved around the question whether there are voids in the Ly $\alpha$ forest, similar in comoving size to those seen in the local galaxy distribution. In principle, verification of the existence of a void large enough to have a vanishing probability of occurring by chance, if drawn from a Poissonian distribution, is straightforward. The probability function for a Poissonian gap of size $\Delta z$ in a spectrum with absorption line density $d \mathcal{N} / d z$ is simply

$$
P(\Delta z)=\exp \left[-\left(\frac{d \mathcal{N}}{d z}\right) \Delta z\right]
$$

Carswell \& Rees (1987) concluded that voids with sizes like those in the local universe $\left[\sim 50 \mathrm{~h}^{-1} \mathrm{Mpc}\right.$ (comoving)] cannot fill more than $5 \%$ of the volume at $\langle z\rangle \sim 3.2$. This result was confirmed by work by Duncan et al (1989), based on a larger data set. A similar conclusion was reached by Pierre et al (1988), who found that the Ly $\alpha$ absorbing gas cannot exhibit a void structure that is similar to low redshift galaxies without producing strong clustering that is inconsistent with the observations.

Nevertheless, individual large gaps have been found. Crotts (1987) discovered a $43 \mathrm{~h}^{-1} \mathrm{Mpc}$ gap toward Q0420-388. This result was variously contested and confirmed in a dispute about significance levels (Duncan et al 1989, Ostriker et al 1988, Crotts 1989, Bi et al 1991, Rauch et al 1992), fuelled by, among other things, the lack of a universally adopted definition of "void," which takes into account that a void may be void of lines only down to a certain detection threshold. It turned out that Crotts' gap, though not entirely empty, is a region of significantly lower line density. Dobrzycki \& Bechtold (1991) found another void that was $\sim 32 \mathrm{~h}^{-1} \mathrm{Mpc}$ in the spectrum of Q0302-003, and Cristiani et al (1995) discovered a significant pair of smaller voids toward Q0055-269. To summarize, the Ly $\alpha$ forest does show the occasional gap, but the void structure apparent in the local galaxy population is not present in Ly $\alpha$ absorbing gas.

The origin of the rare voids has most often been discussed in connection with a local proximity effect: A foreground QSO near the LOS to the more distant QSO produces a "clearing" by ionizing the adjacent clouds seen in the LOS to the other object (Bajtlik et al 1988, Kovner \& Rees 1989). No 
clean-cut evidence has been found for this effect in the few studies done to date (Crotts 1989, Møller \& Kjaergaard 1991), nor has it been possible to rule out its existence (e.g. for the 0302-003 void, see Dobrzycki \& Bechtold 1991, Fernandez-Soto et al 1995). Several effects can complicate the analysis. Short of abandoning the idea of the proximity effect as an excess of ionization caused by a nearby QSO, anisotropic emission (beaming) by the QSO and QSO variability can be invoked to explain the nondetections and the discrepancies between the redshift positions of QSO and candidate voids. The number of free parameters in such models is currently of the same order as the number of voids observed, suggesting that studies of individual voids probably will not be of great use for some time. With a sufficiently large data set, global searches for fluctuations in the absorption pattern caused by an inhomogeneous radiation field (Zuo 1992, Fardal \& Shull 1993) may have a better chance of success. However, Haardt \& Madau (1996) have recently pointed out that the diffuse recombination radiation from Ly $\alpha$ clouds themselves (which can provide on the order of $30 \%$ of the ionization rate) can considerably reduce the fluctuations.

\subsection{Spatial Structure Across the Sky: Multiple Lines of Sight}

The lack of two-dimensional information is one of the main shortcomings of high redshift QSO spectroscopy. This lack makes it hard to understand the geometry of the absorbers and to disentangle positions in velocity and real space. Through observations of common absorption systems in spatially separated LOS (multiple images of gravitationally lensed QSOs or QSO pairs), the spatial dimension(s) across the sky can be restored to some degree. Gravitationally lensed QSOs have maximum image separations up to a few arcseconds, giving information on scales $<100 \mathrm{kpc}$. In contrast, QSO pairs rarely occur closer to each other than a few arcminutes, which limits the scale that can be probed at high redshift to larger than a few hundred kiloparsecs. The presence or absence of common absorption in two LOS gives an indication of the coherence length of the absorber. Obviously, common absorption systems must be at least as large as the transverse separation between the beams to appear in both LOS. If some of the systems are missing in one image, a maximum likelihood estimate based on the binomial probability distribution for the fraction of "hits" and "misses" can be used to estimate the mean extent of the absorbing cloud across the sky (McGill 1990).

GRAVITATIONALLY LENSED QUASISTELLAR OBJECTS The first gravitational lens discovered, the $z=1.39$ QSO Q0957+561 (Walsh et al 1979), enabled Young et al (1981) to put a lower limit of $\sim 7 \mathrm{kpc}$ on the size of an intervening C IV absorption system common to both LOS. Another notorious lensed QSO, 
$2345+007 \mathrm{~A}, \mathrm{~B}$, yielded lower limits to the size of Ly $\alpha$ clouds of $1-11 \mathrm{~h}^{-1} \mathrm{kpc}$ (at $z \sim 2$ ) (Foltz et al 1984, McGill 1990); the uncertainty comes from the unknown position of the lensing object. This scale, though strictly an upper limit, came to be considered as a typical "size" for several years, until the observations of UM $673(z=2.73$; lens at $z \sim 0.5$; LOS angular separation 2.2 arcsec) by Smette et al (1992) showed two virtually identical Ly $\alpha$ forests in the $A$ and $B$ images, with equivalent width and velocity differences consistent with the measurement errors. With proper LOS separations ranging up to $1 \mathrm{~h}^{-1} \mathrm{kpc}$ for Ly $\alpha$ systems, a 2- $\sigma$ lower limit of $12 \mathrm{~h}^{-1} \mathrm{kpc}$ to the diameter of the clouds (which were assumed to be spherical) was obtained. Observations of another bright lensed QSO (HE 1104-1805A,B) by Smette et al (1995) produced even more stringent lower limits to cloud diameters of $25 \mathrm{~h}^{-1} \mathrm{kpc}$. These remarkable results seem to indicate that the $\operatorname{Ly} \alpha$ forest absorbers are not consistent with the relatively small clouds envisaged in the pressure confined model, nor are they consistent with the possibility that the absorption of a significant fraction of the systems could arise in the virialized regions of galaxies or could exhibit the small-scale variations typical of the interstellar medium.

Unfortunately, very few lensed objects are suitable targets for Ly $\alpha$ spectroscopy: At least two images must be bright enough to be observed at high resolution; the image separation must exceed typical seeing conditions ( $>1.5$ arcsec); the emission redshift must be sufficient to shift the Ly $\alpha$ forest into the optical wavelength range. The apparent lack of structure of the forest over several kiloparsecs and the limited angular separation of lensed LOS make observations of QSO pairs more suited for studying the large-scale structure of the Ly $\alpha$ forest proper. Lensed QSOs, however, should become increasingly useful for the study of metal absorption systems and high redshift galaxies, a topic which is beyond the scope of this review (see Smette 1995).

QUASISTELLAR OBJECT PAIRS In view of the crowding in the high redshift Ly $\alpha$ forest and the relatively large separations between most QSO pairs, the crossidentification of individual absorption systems in the two LOS can be difficult, and detections of coherent absorption across the sky may be significant only in a statistical sense. Sargent et al (1982) applied a cross-correlation analysis to the spectra of the QSO pair near 1623+26 (Sramek \& Weedman 1978; also see Crotts 1989, who included two additional QSOs near this pair). With transverse separations $\sim 1-2 \mathrm{~h}^{-1} \mathrm{Mpc}$, the LOS were well-suited for searching for the large coherent absorption pattern predicted by some theories. Oort (1981), for example, had suggested that the Ly $\alpha$ forest was caused by intergalactic gas distributed like low redshift "superclusters." Sargent et al (1982) concluded that there is too little coherence between the systems across the plane of the sky to agree with the large "pancake" structures envisaged by Oort. 
However, evidence for very large structures seen in the much less densely populated C IV forest suggests that some of the coherent absorption in the Ly $\alpha$ forest is missed because of the difficulty of cross-identifying absorbers. Therefore, examples of gaseous structures with large coherence usually have come from high column density systems. Shaver \& Robertson (1983) saw common absorption over $\sim 380 \mathrm{~h}^{-1} \mathrm{kpc}$ at $z \sim 2$ in metal absorption systems (Q0307-195A,B). Francis \& Hewett (1993) found coincident damped Ly $\alpha$ absorption over $\sim 3 \mathrm{~h}^{-1} \mathrm{Mpc}$ across the sky. Considering the firm lower limits from gravitational lensing, there was reason to expect large sizes for the low column density forest as well, and such evidence was eventually found: Optical MMT data of the unusually close QSO pair Q1343+266A,B (also known as Q1343+264A,B; separation of 9.5 arcsec) showed absorbers extending over several hundred kiloparsecs at redshifts just below 2 (Bechtold et al 1994, Dinshaw et al 1994). Even larger sizes appear to occur at somewhat lower redshift. Dinshaw et al (1995) deduced a most probable diameter of $360 \mathrm{~h}^{-1}$ $\mathrm{kpc}$ for $0.5<z<0.9$ from HST FOS spectra of the Q0107-25A,B pair. A later reanalysis points to a median diameter of $\sim 0.5 \mathrm{~h}^{-1} \mathrm{Mpc}$ for Q1343+266 and $\sim 1 \mathrm{~h}^{-1} \mathrm{Mpc}$ for the lower $z$ Q0107-25A,B (Fang et al 1996). The velocity difference between lines in different LOS assumed to belong to the same absorption system, however, are small, with an intrinsic mean difference of only $\sim 50 \mathrm{~km} \mathrm{~s}^{-1}$. Fang et al (1996) found a trend for the size estimate to increase with separation between the LOS, which is indicative of spatial coherence on a range of scales, the upper end of which may not have been sampled yet. Most of these studies assumed spherical clouds, but from photoionization arguments, spherical objects consistent with the measured sizes and column densities can be shown to be so highly ionized as to easily overfill the universe with baryons. The simplest explanation, which allows for a denser and more neutral gas while remaining consistent with the transverse sizes, assumes that typical Ly $\alpha$ clouds are flattened, with a thickness on the order of $\sim 30 \mathrm{~h}^{-1} \mathrm{kpc}$, for transverse sizes of $1 \mathrm{~h}^{-1} \mathrm{Mpc}$ (Rauch \& Haehnelt 1995).

The large sizes, the small velocity and column density differences, the possible absence of a unique size scale, the apparent flatness of the clouds found at high and intermediate redshifts, and the general absence of voids all argue against an origin of the typical (i.e. low column density, $\log N \lesssim 14$ ) Ly $\alpha$ forest line in the potential wells of already formed galaxies.

\section{THE FIRST GENERATION OF MODELS}

Theoretical modeling of absorption systems can be traced back to Spitzer's (1956) prediction (expanded by Bahcall \& Spitzer 1969) that normal galaxies have large gaseous halos giving rise to heavy element UV absorption lines. 
Bahcall \& Salpeter (1965) considered groups of galaxies; Arons (1972) suggested forming low mass protogalaxies as the probable sites of $\mathrm{Ly} \alpha$ absorption. The interpretation by Sargent et al (1980) of their observations of the Ly $\alpha$ forest alerted researchers to differences between metal and Ly $\alpha$ forest absorption systems, with the evidence pointing away from galaxies to distinct astronomical objects: intergalactic gas clouds.

\subsection{Lyman Alpha Clouds Confined by the Pressure of an Intercloud Medium}

If the $\mathrm{Ly} \alpha$ absorbers correspond to overdense clumps of gas, their persistence throughout the history of the universe must be either due to only a slow change in their properties or to replenishment of the clouds on a shorter time scale. An apparent lack of rapid evolution in the properties of the forest (later shown to be a statistical fluke), and the short electron and proton relaxation time scales and mean free paths, appeared to justify treating the clouds as "self-contained entities in equilibrium" (Sargent et al 1980). A two-phase IGM was postulated, with the hot, tenuous intercloud medium (ICM) in pressure equilibrium with the cooler and denser Ly $\alpha$ clouds. The standard version of the pressure confinement model (Sargent et al 1980, Ostriker \& Ikeuchi 1983, Ikeuchi \& Ostriker 1986) considers spherical and, since gravity is ignored, homogeneous clouds. This model is self-consistent, but there are no very compelling physical reasons for preferring pressure to gravitational confinement or to no confinement at all; the possibility of self-gravitating clouds as an alternative was discussed (Melott 1980, Black 1981).

Nevertheless, the pressure confinement model for Ly $\alpha$ clouds is appealing for several reasons: It combines the concept of a multiphase structure of the IGM, which is familiar from the interstellar medium, with the idea of separate entities, "clouds," in analogy to but different from galaxies. A hot ICM may have been a possible source of the X-ray background. In addition, the explosion scenario (see below) provided a theory of cloud formation. Finally, the model made testable predictions, a rare but risky undertaking for astrophysical theories, which paved the way to its eventual demise.

The basic properties of pressure confined clouds, as worked out in detail by Sargent et al (1980), Ostriker \& Ikeuchi (1983), and Ikeuchi \& Ostriker (1986), can be summarized as follows. The Ly $\alpha$ clouds are supposed to be in photoionization equilibrium with an ionizing UV background. The gas is heated by photoionization and cools via thermal bremsstrahlung, Compton cooling, and the usual recombination and collisional excitation processes. The cloud evolution consists of several phases, depending on the relative lengths of cooling and expansion time scales. The ICM is expanding adiabatically by the cosmic expansion at all times because the high degree of ionization does not allow for 
efficient photoionization heating. The denser clouds embedded in the hot ICM start out in isothermal expansion with a temperature fixed by thermal ionization equilibrium $\left(T_{c} \sim 3 \times 10^{4} \mathrm{~K}\right)$, until the density $n_{c} \propto P_{\mathrm{ICM}} / T_{c} \propto(1+z)^{5}$ has dropped sufficiently that photoheating cannot compensate for the work of expansion any longer, and the clouds begin to cool and to expand less rapidly. The sound speed drops even faster, so ultimately pressure equilibrium with the ICM ceases and the clouds enter free expansion. The available range of cloud masses is constrained by the requirements that the clouds must be small enough not to be Jeans-unstable but large enough not to be evaporated rapidly when heated by thermal conduction from the ambient ICM (Sargent et al 1980, Ostriker \& Ikeuchi 1983). Clouds formed at $z \sim 6$ would survive down to accessible redshifts $(\sim 4)$ only if their masses range between $10^{5}<M_{c}<10^{10} \mathrm{M}_{\odot}$.

THE ORIGIN OF LYMAN ALPHA CLOUDS FROM COSMIC SHOCKS The explosion scenario of structure formation (Schwarz et al 1975, Ostriker \& Cowie 1981) provided a nongravitational origin for the pressure confined Ly $\alpha$ clouds. Largescale explosions from galaxies (e.g. from starbursts) and QSOs may have driven shock waves into the IGM. These events may also have provided the energy for collisional reionization and heating of the ICM, since photoionization cannot produce temperatures high enough to maintain pressure confinement (Ikeuchi \& Ostriker 1986). This way a two-phase medium of hot "cavities" enclosed by a system of cooler shells could have arisen (Ozernoy \& Chernomordik 1976). Ly $\alpha$ absorption is caused by the fragmenting shells (Chernomordik \& Ozernoy 1983, Vishniac et al 1985). Among the observational consequences may be pairs of Ly $\alpha$ lines that occur wherever the LOS intersects an expanding, spherical shell. It has been argued that such pairs have been seen (Chernomordik 1988 and references therein); this may be the case in individual systems, but it is difficult to prove in a statistical sense because the two-point correlation does not show the expected signal at the relevant velocity scale, $\sim 100 \mathrm{~km} \mathrm{~s}^{-1}$ (Rauch et al 1992). A potential problem for this model is implied by the observed lack of a correlation with galaxies. If Ly $\alpha$ clouds are shells expelled by galaxies, the absorbers should be clustered in a manner similar to that of galaxies (Vishniac $\&$ Bust 1987). Neither the auto-correlation function among absorbers nor the cross-correlation with galaxies shows a signal of the requisite strength (Barcons \& Webb 1990).

A similar pattern of shell formation ensues if QSOs, in the initial event of reionization, surround themselves with Strömgren spheres (Arons \& McCray 1969, Shapiro \& Giroux 1987), which can lead to shocked shells of gas at the boundary of the H II regions. The shells fragment as in the explosion scenario and may be visible as Ly $\alpha$ absorbers (Madau \& Meiksin 1991).

THE ELUSIVE INTERCLOUD MEDIUM The need for a confining ICM has led to a number of searches for a residual absorption trough between the absorption 
lines, caused by the $\mathrm{H} \mathrm{I}$ in the intercloud space. These measurements came to be referred to in the literature as the Gunn-Peterson (GP) test, though Gunn \& Peterson's (1965) original result of a $40 \%$ average absorption was a detection of the unresolved Ly $\alpha$ forest as a whole. Values for the residual (i.e. intercloud, or diffuse, as opposed to line) GP effect require ( $a$ ) a precise knowledge of the unabsorbed QSO continuum level and $(b)$ either subtracting the contribution from Ly $\alpha$ "lines" or the use of line free regions (whatever that may be) for the estimate. Steidel \& Sargent (1987b) measured the total flux decrement of a sample of eight QSOs against continua extrapolated from the regions redward of Ly $\alpha$ emission. After subtracting a model population of discrete Ly $\alpha$ lines, they obtained a residual $\tau_{G P}<0.02 \pm 0.03(\langle z\rangle \sim 2.67)$, i.e. a null result. Giallongo et al $(1992,1994)$ have compared apparently line free regions with an extrapolated continuum, and they found $\tau_{G P}<0.013 \pm 0.026(\langle z\rangle \sim 3)$ and $\tau_{G P}<0.02 \pm 0.03(\langle z\rangle \sim 4.3)$, respectively. Given the line crowding at high redshift, the small error bars of the $z>4$ work betray a certain degree of optimism.

The clean-cut decomposition into line and continuum absorption makes theoretical sense for pressure confined clouds, but observationally, we can never be sure whether there is a flat continuum trough from diffuse gas or whether there are many weak lines blended together. Jenkins \& Ostriker (1991) and Webb et al (1992), acknowledging this problem, attempted to model the pixel intensity distribution with continuum absorption and variable contributions from discrete lines. Their results show that $\tau_{G P}$ can be produced both ways, by blending of weak lines below the detection threshold or by a constant pedestal of absorption.

In any case, the weakness or nondetection of a residual GP trough puts an upper limit on the density of the ICM. A lower limit on the ICM pressure $\left(\propto n_{\mathrm{ICM}} T_{\mathrm{ICM}}\right)$ can be derived from the absorption line width (which gives an upper limit on the radius of the expanding cloud) (Ostriker \& Ikeuchi 1983). The condition that the cloud must be large enough not to evaporate gives an upper limit on the pressure. Another independent upper limit on the pressure of the ICM comes from the lack of inverse Compton distortions in the spectrum of the cosmic microwave background (Barcons et al 1991). This result rules out the IGM as the source of the hard X-ray background. It also may spell trouble for the explosion model of galaxy formation, which is the origin of the two-phase IGM in the current picture, and of apparently nonexisting structure in the cosmic microwave background. When all the limits are combined, only a relatively small corner of allowed $(n, T)$ parameter space remains for the ICM.

PROBLEMS WITH THE COLUMN DENSITY DISTRIBUTION For pressure confined clouds, the large range of neutral hydrogen column densities observed must correspond to a range in the parameter combination

$$
N(\mathrm{H} \mathrm{I}) \propto M_{c}^{1 / 3} T_{c}^{-29 / 12} J^{-1} P^{-5 / 3} .
$$


To reproduce only the low column density systems between $13<\log N(\mathrm{H} \mathrm{I})$ $<16$, the mass has to vary by 9 orders of magnitude, the radiation field by 3 orders, or the pressure by a factor of 63 . To ensure cloud survival, the mass range is limited to less than 4 dex (see above) and the temperature is constant; therefore, we may need to invoke pressure inhomogeneities (Baron et al 1989). However, Webb \& Barcons (1991), looking for pressure-related spatial correlations among the equivalent widths of Ly $\alpha$ forest lines, excluded pressure fluctuations $\Delta P / P>14 \%$ at the $2-\sigma$ level, and a similar limit must hold for the radiation field $J$. Extremely flattened clouds would help somewhat in that they would increase the column density range, allowing a wider range of path lengths through the clouds (Barcons \& Fabian 1987), but that may introduce other problems. Williger \& Babul (1992), taking these constraints into account, investigated pressure confined clouds with detailed hydrodynamical simulations and found that the small mass range leads not only to a failure in producing the column density range but also to a faster drop in the number of clouds with redshift than observed.

To summarize, the pure pressure confinement model is unlikely to explain the Ly $\alpha$ forest as a whole, though it is clear that some LOS must go through sites where gas is locally confined by external hydrostatic or ram pressure. Low redshift gaseous galactic halos, the likely hosts of the dense Lyman limit absorbing clouds, may be such environments. Formed by local instabilities, the dense clouds may be in pressure equilibrium with a hot gas phase at the virial temperature of the halo (Mo \& Miralda-Escudé 1996 and references therein).

\subsection{Gravitational Confinement: Self-Gravity}

Self-gravitating baryonic clouds were suggested by Melott (1980) as an alternative to the pressure confinement model. Black (1981) investigated in detail the physical structure of such objects. He found that (quasi)stable clouds with properties consistent with the observations have to be extended $(\sim 1 \mathrm{Mpc})$ and must either be truncated by an external medium or be large enough to overlap, providing their own boundary pressure. In this model, the appearance of the IGM as a forest of lines is more due to the strong internal gradients of the neutral gas density than to a sharp transition between separate entities. The density of the IGM along the LOS undulates, and there is no real difference between an ICM and the clouds. The huge sizes would also have been able to reconcile a larger mass density of the IGM with the observed cloud parameters, whereas pressure confined clouds would contain only a small fraction of all baryons. The model met with scepticism because the large sizes appeared to contradict the (then scant) observational evidence. However, it may also have other problems, such as reproducing the column density distribution (Petitjean et al 1993a). 


\subsection{Gravitational Confinement: Clouds Dominated by Hot Dark Matter Gravity}

The advent of ab initio theories of gravitational structure formation made it possible to place the Ly $\alpha$ forest in a larger frame and investigate its relation to galaxy formation. In principle, the number density, sizes, and physical parameters of the absorbers can be predicted as a consequence of cosmological models, though until recently, this has been wishful thinking.

ZELDOVICH PANCAKES The first such theory to explicitly address the Ly $\alpha$ forest phenomenon was the hot dark matter (HDM) model. The formation of adiabatic pancakes, suggested by Zeldovich (1970), is expected to produce a primordial gas phase with the right properties to show up in $\mathrm{H}$ I absorption (Doroshkevich \& Shandarin 1977). Among the interesting consequences of this theory are the large (in fact, too large) sizes of the pancakes. Even after fragmentation, coherent absorption should be extending over megaparsecs across the sky (Doroshkevich \& Muecket 1985), thus explaining the large transverse sizes seen later in QSO pair studies. In a prescient paper, Oort (1981), at the time referring to the HDM pancakes, suggested identifying Ly $\alpha$ absorbers with collapsed but uncondensed gas in the "superclusters" of galaxies known from low redshift. He calculated the gravitational scale height of a sheet of gas and noted the similarity of the mean free path between Ly $\alpha$ clouds and the distances between superclusters. The very large transverse sizes expected $(\sim 20 \mathrm{Mpc}$, at $z \sim 2$ ) were not confirmed, however, by the QSO pair study of Sargent et al (1982), so such objects cannot be the rule.

The underlying structure formation scenario (HDM) has become somewhat unpopular, but the physical idea of Ly $\alpha$ absorbers as flattened pancakes survives into the currently favored CDM-based picture (see below). However, the CDM pancakes (or sheets) are more than an order of magnitude smaller than the Zeldovich ones, and they form late, after denser structures like knots and filaments are already in place (Cen et al 1994, Bond et al 1996).

\subsection{Gravitational Confinement: Cold Dark Matter Minihalos}

The properties of gas clouds under the influence of the gravitational field of dark matter have been investigated by Umemura \& Ikeuchi (1985) and more specifically in terms of the "minihalo" model by Rees (1986) and Ikeuchi (1986). In this picture, Ly $\alpha$ clouds are a natural byproduct of the CDM structure formation scenario. Photoionized gas settles in the potential well of an isothermal CDM halo. The gas is stably confined if the potential is sufficiently shallow to avoid gravitational collapse but deep enough to prevent the warm gas from escaping. CDM minihalos are more compact than the self-gravitating baryonic clouds of Black (1981) because of the larger dark matter gravity. The detailed structure 
of the halo depends on the relative spatial distribution of baryons and CDM. The models can be parametrized by the intensity of the radiation field $J$, the central overdensity $\delta(r=0)$, and the ratio of baryonic to dark matter (Ikeuchi et al 1988). The minihalo model has the attractive feature of providing a natural explanation for the overall shape of the observed column density distribution function (CDDF). The large observed dynamic range in column density reflects the strong density variations as a function of impact parameter, rather than a range in cloud properties. For the general case where the baryon distribution is an isothermal sphere $\left(n_{b} \propto r^{-2}\right)$, the $\mathrm{H} \mathrm{I}$ density in the highly ionized region of the minihalo drops like $n_{\mathrm{H} \mathrm{I}} \propto r^{-4}$ with radius $r$, and the resulting column density distribution seen by random LOS through a population of such halos obeys $d \mathcal{N} / d N_{\mathrm{H} \mathrm{I}} \propto N_{\mathrm{H} \mathrm{I}}^{-1.5}$ (Milgrom 1988, Rees 1988). The largest column densities, including damped Ly $\alpha$ systems, are caused by the neutral cores in the shielded centers of the clouds (Murakami \& Ikeuchi 1990). Thus minihalos can produce a column density power law over almost nine decades, providing a physical basis for Tytler's (1987a) suggestion of a common origin for all QSO absorbers. Evolution with redshift is caused by a number of processes (Rees 1986): When loosing pressure support as the ionizing flux decreases, gas may settle deeper into the potential well, thus reducing the geometric absorption cross section. Once stars are forming, the UV flux may rise again, and stellar winds may blow out the gas (Babul \& Rees 1992), thus increasing the absorption cross section. Halos are produced by a continuing turnaround of density peaks and are destroyed by merging.

A nonstationary version of the minihalo model was studied by Bond et al (1988), who examined the hydrodynamics of a collapsing spherical top-hat perturbation. If the accreting baryonic component escapes gravitational collapse (and subsequent star formation), it may reexpand under the influence of photoionization heating and even recollapse after the UV intensity has ebbed (see also Murakami \& Ikeuchi 1993).

In order to investigate the relative importance of various confinement mechanisms, Petitjean et al (1993a) have studied a hybrid model of spherical clouds, with or without dark matter, bounded by an external pressure. These models show that pure baryonic clouds (as discussed by Black 1981), in order to be stable against outer pressure, tend to overproduce high column density systems. Many of the observed features of the Ly $\alpha$ forest clouds can be explained with a single type of minihalo, but to match the fine structure of the CDDF, halos may be required to exhibit a range of central densities (Murakami \& Ikeuchi 1990, Petitjean et al 1993b). Two separate populations, one for low column density Ly $\alpha$ clouds and one for the higher column density metal systems, give a better fit to the CDDF (Petitjean et al 1993b).

Charlton et al $(1993,1994)$ have studied gravitational condensations with a different geometry, modeling Ly $\alpha$ clouds as equilibrium slab models that are 
subject to the pull of CDM gravity and to an external pressure. It was found that the change of slope in the CDDF (near $\log N(\mathrm{H} \mathrm{I}) \sim 15$ ) can be explained by a transition between pressure and gravitational confinement, in the sense that at higher column densities gravity takes over and imposes a steeper dependence of the neutral column density with total column density.

NONEQUILIBRIUM AND OTHER EFFECTS POINTING BEYOND THE SIMPLE HALO MODEL With the adoption of the CDM-based models, researchers could avail themselves of the analytical apparatus developed to describe the dark matter distribution, especially the important concept of "halos" (Babul 1990, Mo et al 1993). Of course, there is a limit to the degree of realism with which a counting scheme for dark matter condensations or spherical collapse can describe the observed properties of gas clouds, and even the notion of distinct "objects," dear to traditional astronomy, may fail. Hydrodynamic simulations (see below) show that in a hierarchical universe at intermediate redshift $(\sim 2)$, most baryonic matter may not have settled in spherical or rotationally supported virialized objects, as suggested by the word halo. Virial radii of objects capable of stably confining H I clouds are $\sim 10 \mathrm{kpc}$ (Rees 1986). The coherence lengths of Ly $\alpha$ systems from gravitational lensing constraints (Smette et al 1992, 1995) are much larger, implying that only few LOS ever hit the virialized region. Thus minihalos may not only be embedded in regions of uncollapsed gas; they may still be accreting matter at the epoch where we observe the Ly $\alpha$ forest. Thus it makes sense to look for signs of nonequilibrium, especially departures from thermal line profiles caused by the bulk motion of the infalling gas (MiraldaEscudé \& Rees 1993). Meiksin (1994) has traced the formation and internal structure of minihalos and slabs with hydrodynamical simulations to search for such observable nonequilibrium effects. For a slab or pancake geometry, noticeable deviations from Voigt profiles are predicted, but they would be hard to detect for spherical clouds.

\section{THE COSMOLOGICAL SIGNIFICANCE OF THE LYMAN ALPHA FOREST}

Over the past few years, semianalytical work and in particular hydrodynamic simulations of hierarchical structure formation have gradually led to a minor Copernican shift in our perception of the material content of the high redshift universe. If the inferences (discussed below) are interpreted correctly, the IGM is the main repository of baryons down to redshifts at least as low as $z \sim 2$. If so, then high redshift galaxies-in absorption line parlance, "Lyman limit" or "damped Ly $\alpha$ systems"- -are mere tracers of the matter distribution. The simulations show that the Ly $\alpha$ forest is produced by a hierarchy of gaseous structures, with typical shapes changing from sheets through filaments to spherical 
galactic gaseous halos, as the column density increases. Perhaps most important, Ly $\alpha$ forest lines closely reflect gravitationally induced density fluctuations in the general matter distribution. Given the relatively simple physics of this baryonic reservoir and the enormous sensitivity of the observations, Ly $\alpha$ forest spectra should make excellent and largely unbiased probes of structure formation at high redshift.

THE LARGE BARYON CONTENT The fraction of matter incorporated into galaxies or still left in the IGM depends strongly on the structure formation model. To calculate the baryon content of Ly $\alpha$ clouds, we need to know the ionization correction, as most of the gas is highly ionized. For a given ionizing radiation field, the degree of ionization depends on the density and thus, for a given observed column density, on the spatial extent of the gas. Deriving the mass content then requires fixing the size (or scale height) of the clouds either from measurement or from theoretical prejudices. For example, the fraction of mass required to cause the observed amount of absorption can be quite large for gravitationally confined, extended, baryonic clouds (Black 1981). In contrast, the small baryon content expected if the Ly $\alpha$ were caused by pressure confined clouds (Sargent et al 1980) is largely a result of the small cloud sizes adopted. By using a suitable choice of parameters, the Ly $\alpha$ forest can be made to contain anything, from a negligible fraction up to virtually all of the baryons, and still be consistent with the observations (Meiksin \& Madau 1993). Specifically, for the CDM minihalo model, Petitjean et al (1993b) found that the Ly $\alpha$ forest clouds had to contain most of the baryons at redshift 2-3 in order to match the observed CDDF. This is in agreement with Shapiro et al (1994), who found that in a CDM model, the fraction of baryons not yet collapsed into galaxies should be on the order of 50-90\%. Independent of the cosmological model, the large transverse sizes of Ly $\alpha$ absorbers measured from QSO pairs give another indirect indication that the baryon density in Ly $\alpha$ clouds must be large or the absorbers must be extremely flattened (Rauch \& Haehnelt 1995).

A FOREST OF LINES, OR A FLUCTUATING GUNN-PETERSON EFFECT? Under the influence of gravity, the IGM becomes clumpy and acquires peculiar motions, and so the Ly $\alpha$ (or GP) optical depth should vary even at the lowest column densities (Black 1981, McGill 1990, Bi et al 1992, Miralda-Escudé \& Rees 1993, Reisenegger \& Miralda-Escudé 1995). In a CDM-dominated structure formation scenario, the accumulation of matter in overdense regions reduces the optical depth for Ly $\alpha$ absorption considerably below the average in most of the volume of the universe, leading to what has been called the fluctuating GP phenomenon. Traditional searches for the GP effect that try to measure the amount of matter between the absorption lines are no longer very meaningful, as 
they are merely detecting absorption from matter leftover in the most underdense regions. If this is not taken into account, the amount of ionizing radiation necessary to keep the neutral hydrogen GP absorption below current detection limits easily can be overestimated.

As another consequence, the distinction between the low column density Ly $\alpha$ forest "lines" and the GP "trough" becomes somewhat artificial. Bi and collaborators (Bi et al 1992, Bi 1993, Bi \& Davidsen 1997) have shown that the optical depth fluctuations corresponding to the linear regime of gravitational collapse in the IGM can give a remarkably realistic representation of the Ly $\alpha$ forest (ignoring the higher column density lines, which are produced from nonlinear structures, e.g. minihalo type objects). Their semianalytical work is based on a log-normal density fluctuation field. For low densities where dissipation is not important, the collapse of dark matter and baryons differs mainly by the presence of the gas pressure, which effectively smooths the baryon distribution on scales below the Jeans length. Bi and coworkers treated the pressure as a modification to the power spectrum of the baryon density contrast $\delta_{b}$, suppressing power on scales below the Jeans length:

$$
\delta_{b}(k)=\frac{\delta_{D M}(k)}{1+k^{2}\left(\lambda_{J} / 2 \pi\right)^{2}},
$$

where $\lambda_{J}$ is the Jeans length, $k$ the wavenumber, and $\delta_{D M}$ the dark matter overdensity. This method can elucidate many of the basic features of low column density Ly $\alpha$ clouds. The schematic treatment of the equation of state and the lack of inclusion of shock heating limit the approach, however, to overdensities of $\delta<5$, where gas physics beyond the Jeans criterion is not very important.

\subsection{Hydrodynamic Simulations of the Lyman Alpha Forest}

NUMERICAL APPROACHES From the early 1990s on, hydrodynamic cosmological simulations became sufficiently realistic to be able to quantitatively predict the physical properties of the IGM and the high redshift Ly $\alpha$ forest from the initial conditions of a given structure formation model (e.g. Cen \& Ostriker 1993). A Ly $\alpha$ forest spectrum is specified completely by the Hubble constant, gas density, temperature, peculiar velocity, and neutral fraction along the LOS. By predicting these quantities for artificial QSO LOS through simulated slices of the universe, it becomes possible to examine the correspondence between Ly $\alpha$ forest absorbers and the physical properties of the underlying gaseous structures. This approach was first taken by Cen et al (1994; see also Miralda-Escudé et al 1996) using a Eulerian hydrosimulation of a CDM model with a nonzero cosmological constant $\Lambda(\Lambda \mathrm{CDM})$. Since then, a range of other numerical techniques has been applied to different cosmological models. The basic properties of the Ly $\alpha$ forest turn out to be only weakly dependent on the cosmological 
model, and similar answers have been obtained with a variety of approaches: Petitjean et al (1995) grafted the baryons onto a COBE-normalized CDM particle mesh simulation, using an analytic prescription to track the thermal history of the gas. A standard CDM model has been studied by Zhang et al (1995, 1997) with a Eulerian code and by Hernquist et al (1996) with a Lagrangian, smoothed particle hydrodynamics (SPH) technique. As a crude general rule, the Eulerian codes are capable of higher resolution for the void regions producing the lowest column density Ly $\alpha$ forest, whereas the Lagrangian codes are superior for regions like minihalos or galaxies where a larger dynamic range is required. Thus the use of SPH codes has been extended to study damped Ly $\alpha$ systems (Katz et al 1996) and metal absorption systems (Haehnelt et al 1996b). Hybrid schemes (e.g. Wadsley \& Bond 1997) can be tailored to capture the influence of both large-scale (long wavelength) gravitational effects and the small-scale gas dynamics on the formation of $\operatorname{Ly} \alpha$ absorbers.

THE NATURE OF LYMAN ALPHA ABSORBERS In spite of some quantitative differences, a generic picture of the Ly $\alpha$ forest has emerged from these studies: Low column density systems $[\log N(\mathrm{H} \mathrm{I}) \lesssim 14]$ are associated with sheet-like structures, not unlike small versions (length scale of about a few hundred kiloparsecs to $\sim 1 \mathrm{Mpc}$ proper) of Zeldovich pancakes. Gas accretes through weak shocks (creating a double humped temperature profile) and settles in a dense, central cooling layer, presumably to form stars. At the lowest column densities, gas remains unshocked and just bounces back because of the hydrostatic pressure. The gas is partly confined by gravity and partly by ram pressure. Higher column density clouds arise in more filamentary structures, with column density contours of $\log N(\mathrm{H} \mathrm{I}) \sim 14$ extending continuously and with relatively constant thickness ( 40-100 kpc proper) over megaparsec distances. With increasing column density, the absorber geometry becomes rounder; column density contours at $\log N(\mathrm{H} \mathrm{I}) \gtrsim 16$ invariably are spherical, entering the regime where the absorbers more closely correspond to minihalos; there the enclosed gas column is high enough to make the absorption system appear as a Lyman limit or damped Ly $\alpha$ system. Figure 2 shows the spatial appearance of the Ly $\alpha$ absorbers. The visual appearance of the low column density, sheet-like filamentary structure has been aptly described as a "Cosmic Web" (Bond \& Wadsley 1997). Looking at the higher column density, optically thick gas on scales of several megaparsecs gives a somewhat different impression of chains of mini- or larger halos, lining up like pearls on a string, quite similar to the structure seen in $\mathrm{N}$-body simulations of the dark matter distribution. Confirming earlier analytical work, a large fraction of all baryons (80-90\%) resides in the low column density $\operatorname{Ly} \alpha$ forest, mostly in the column density range $14<\log N(\mathrm{H} \mathrm{I})<15.5$ (Miralda-Escudé et al 1996). 


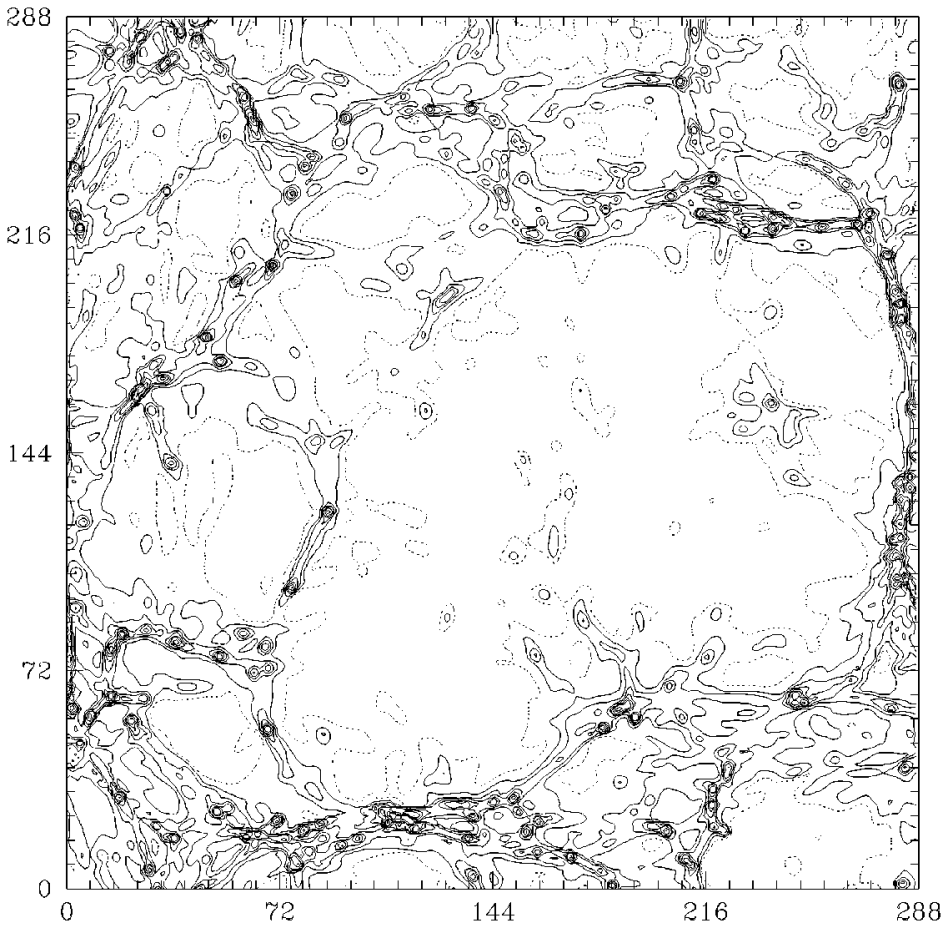

Figure $2 \mathrm{H}$ I column density contours for a slice of the $10 \mathrm{~h}^{-1} \mathrm{Mpc}$ (comoving) box from a cold dark matter model with a nonzero cosmological constant $\Lambda(\Lambda \mathrm{CDM})$ by Miralda-Escudé et al (1996).

A glance at a typical density-temperature diagram (Figure 3) for random LOS through one of the SPH simulations (Haehnelt et al 1996a) reveals significant departures from thermal photoionization equilibrium for all but the highest density gas $\left(n_{H}<10^{-3} \mathrm{~cm}^{-2}\right)$. The temperature-density relation is generally steeper than the equilibrium curve because the lower density gas cools by expansion, while the gas in the density range $n_{H} \sim$ a few times $10^{-5}-10^{-3}$ $\mathrm{cm}^{-3}$ is heated by adiabatic compression or shock heating. Temperatures below $10^{4} \mathrm{~K}$ occur in voids where the expansion velocity is largest.

The gas is still being accreted at the epoch of observations $(z \sim 3)$. Nevertheless, the lower column density, flattened gas structures expand in proper coordinates because the gravitational pull decreases together with the dark matter surface density as the universe expands. Many of the weaker absorption lines arise in low density, relatively extended regions, which expand with a substantial fraction of the Hubble velocity. The expansion and the low temperatures 


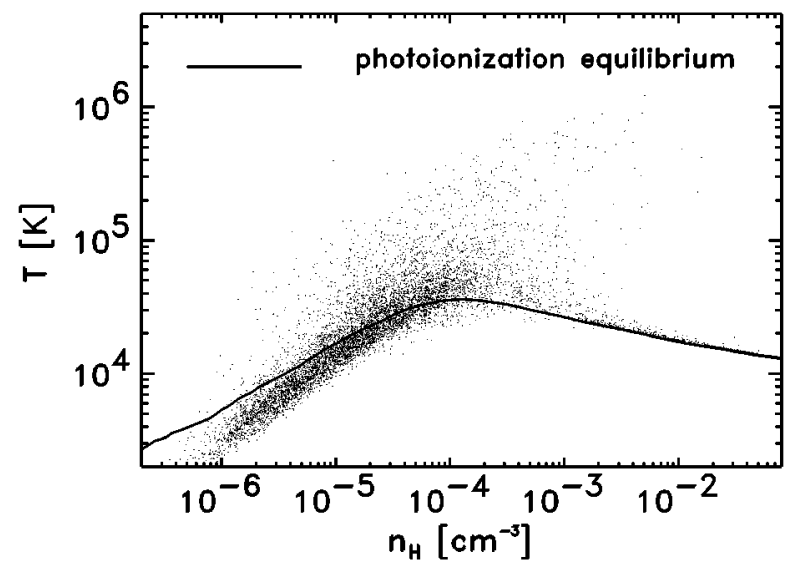

Figure 3 Density-temperature ( $n$ - $T$ ) diagram of the Ly $\alpha$ forest at redshift 3.1 from a smoothed particle hydrodynamics (SPH) simulation of a standard CDM universe (Haehnelt et al 1996a). Each dot represents the mean values of the total hydrogen density $n$ and the gas temperature $T$ along a random line of sight (LOS) through the simulated box. The solid curve gives the locus of thermal photoionization equilibrium. Departures from this curve are due to the dynamical nature of the universe. For all but the most dense regions, expansion cooling in voids (low density regions) and heating by compression and shocks during gravitational collapse steepen the $T(n)$ relation compared to the equilibrium curve.

due to the low density and the adiabatic cooling in voids ensure that at column densities $\log N(\mathrm{H} \mathrm{I}) \lesssim 13$, bulk motion becomes the dominant source of line broadening (Miralda-Escudé et al 1996, Weinberg et al 1997).

MATCHING THE OBSERVATIONS The simulations have been quite successful in matching the overall observed properties of the absorption systems, and the agreement ranges from the acceptable to the amazing. The shape of the column density distribution and the Doppler parameter distribution are reasonably well reproduced by the simulations (Cen et al 1994, Zhang et al 1995, 1997, Hernquist et al 1996, Miralda-Escudé et al 1996). Although the approximate range of Doppler parameters is hard to miss (with photoionization being the great equalizer), subtle effects can raise or lower the mean line width by $\sim 30 \%$ and change the shape of the Doppler parameter distribution. There may be some discrepancy for the Doppler parameters between different simulations (Miralda-Escudé et al 1996, Davé et al 1997, Zhang et al 1997), but it is not yet clear whether this is due to different types of data analysis, different assumptions about the process of reionization, or limited numerical resolution. A departure from Voigt profile shapes, especially the broad wings of weak lines signifying bulk motion broadening in sheets, is seen in the simulations (Cen et al 1994) and 
appears to be present in real high resolution spectra (Rauch 1996). The large transverse sizes of the absorbers seen against background QSO pairs and lensed QSOs are readily explained by the coherence length of the sheets and filaments (Miralda-Escudé et al 1996, Cen \& Simcoe 1997, Charlton et al 1997). The weak clustering amplitude appears to be in agreement with the observations. The histogram of residual fluxes in the Ly $\alpha$ forest is reproduced very well by the models (Rauch et al 1997). Conversely, we may take this as observational evidence in favor of some sort of hierarchical structure formation.

The evolution of the Ly $\alpha$ forest with time at high redshift is mainly driven by the Hubble expansion and the resulting increase in the mean ionization of the gas and to a lesser degree by the gas streaming along the filaments (MiraldaEscudé et al 1996). Muecket et al (1996), from their simulation, found that the number of absorbers per redshift is given by a broken power law, with $\gamma$ $\sim 2.6(1.5<z<3)$ and $\gamma \sim 0.6(0<z<1.5)[\log N(\mathrm{H} \mathrm{I})>14]$ (Riediger et al 1998), in remarkable agreement with the observed data. The break in the power law can be understood as a change with time in the dimensionality of the structures dominating the absorption. The sheet-like absorbers dominating the high redshift Ly $\alpha$ forest are expanding with time and are dropping below the detection threshold first because of their low column density, leaving the absorption from the less rapidly evolving gas distribution in the filaments and knots to dominate. There the column density also decreases, but since the original column was higher, the filaments remain visible for longer. Continuing infall also contributes to the increasing prominence of the more compact structures.

\subsection{The Lyman Alpha Forest as a Cosmological Laboratory}

The first generation of simulations was largely aimed at establishing the physical properties of the Ly $\alpha$ absorbers. The newly gained understanding of the nature of the Ly $\alpha$ forest and the increasing realism of the simulations, together with new semianalytic methods and novel ways of data analysis, have brought quantitative cosmology with the low column density Ly $\alpha$ forest within reach.

Aside from the cosmic microwave background, the IGM is the only astrophysical environment for which the observable properties can (at least in principle) be calculated from a simple set of cosmological initial conditions. This is because at $z \sim 3$, the density fluctuations $\delta=\rho / \bar{\rho}-1$ on spatial scales relevant for detectable Ly $\alpha$ lines (on the order of $10^{2} \mathrm{kpc}$ comoving) are not too far into the nonlinear regime, so the history of the gas causing most of the low column density Ly $\alpha$ forest has not yet been obliterated by virialization and dissipative processes. Overdensities between $\delta \lesssim 0$ and $\delta \sim 15$ roughly correspond to Ly $\alpha$ lines on the linear part of the curve of growth $(12 \lesssim \log N \lesssim 14)$, where spectroscopic measurements are most sensitive. When observing structures 
still dominated by gravity, the problem of "bias," one of the main obstacles to doing cosmology with galaxies, largely can be avoided.

The link between the observable appearance of the Ly $\alpha$ forest and the various cosmological input parameters can be described by the GP relation for the H I optical depth, generalized to include an inhomogenous density and velocity field. As long as the gas is highly ionized and in photoionization equilibrium (not necessarily thermal equilibrium), and the gas is unshocked, the optical depth is given by

$$
\begin{aligned}
\tau= & \frac{0.091}{\Gamma_{-12}}\left(\frac{\Omega_{b} h_{50}^{2}}{0.05}\right)^{2}\left[\frac{5.2 H(0)}{h_{50} H(z)}\right] T_{4}^{-0.7}\left(\frac{\rho}{\bar{\rho}}\right)^{\alpha}\left(\frac{1+z}{1+2}\right)^{6} \\
& \times\left[1+\frac{d v_{p e c}}{H(z) d r}\right]^{-1} .
\end{aligned}
$$

This equation relates the optical depth for Ly $\alpha$ absorption to the mean baryonic density (in gas) in units of the critical density, $\Omega_{b}$, the Hubble constant at redshift $z, H(z)$, the average gas temperature $T$, the proper baryon density $\rho$, the photoionization rate $\Gamma_{-12}$ in units of $10^{-12} \mathrm{~s}^{-1}$, and the gradient of the local peculiar velocity $d v_{p e c} / d r$ along the LOS. A further convolution with a Voigt profile is necessary to include the proper thermal velocity broadening. To turn this relation into a complete description of the observed Ly $\alpha$ forest, cosmology has to predict the cosmic density and velocity fields, the fraction of the closure density in the form of gas, the equation of state, $T=T(\rho)$, and the ionizing radiation field. The exponent $\alpha$ ( $\alpha=2$ for an isothermal gas) takes account of the fact that in denser regions of the universe, the gas is typically warmer because it is more effectively heated by photoionization, but $\alpha$ also depends on the reionization history of the gas and the amount of adiabatic expansion/compression. Hui \& Gnedin (1997) and Croft et al (1997) found values of $\alpha \approx 1.6-1.8$.

Cosmological parameters can now be "measured" by iterating simulations with different input parameters until the simulated statistics of $\tau(z)$ (the mean absorption, correlation function, etc) match the observed ones. Perhaps the simplest cosmological parameter combination to be obtained is related to the baryon density, $\Omega_{b}$. Given Equation 17, the optical depth $\tau$ scales approximately with $\left(\Omega h_{50}^{2}\right)^{\alpha} / \Gamma_{-12}$. Using an independent estimate of the photoionization rate $\Gamma_{-12}$, e.g. from the integrated UV radiation of QSOs, $\Omega_{b}$ can be determined.

The simulations show that a rather high $\Omega_{b} h^{2}$ is required to reproduce the amount of observed absorption, even for a conservatively low estimate of $\Gamma$ (Hernquist et al 1996, Miralda-Escudé et al 1996). If the ionizing background is given by the known QSOs alone and a Haardt \& Madau (1996) spectrum 
(scaled to $J_{-21} \approx 0.23$, at $z=2$ ) is adopted, $\Omega_{b} h^{2}>0.017$ (Rauch et al 1997). Although the observations of $\tau_{\text {eff }}$ on which the result is based are still somewhat uncertain, relatively large $\Omega_{b}$ values appear to be an inherent feature of hierarchical structure formation and cannot be avoided unless most of the low column density absorption dominating the Ly $\alpha$ forest has a different physical origin (Weinberg et al 1997).

COSMOLOGICAL PARAMETERS WITHOUT HYDRODYNAMICS Rerunning the simulations with different input parameters and/or evolving them over a long time span is expensive, as is the analysis of large observed and simulated data sets. A number of new semianalytical techniques have been developed to avoid these difficulties. Although not "hydrodynamically correct," they can give new insights into important aspects of the sometimes obscure dependence of the observational properties on the underlying physical environment. Such techniques compensate for the absent hydrodynamics by various analytical recipes: Petitjean et al (1995) and Croft et al (1998) used dark matter simulations and assumed that baryons (furnished with a suitable analytical thermal history) trace the dark matter directly; Bi et al (1992) and Hui et al (1997) applied power spectra with various forms of cutoff to mimic the smoothing introduced by the Jeans length; Gnedin \& Hui (1997), using a dark matter code, simulated the effects of the gas pressure by modifying the gravitational potential.

THE LYMAN ALPHA FOREST AS A RECORD OF PRIMORDIAL FLUCTUATIONS Semianalytical work by Gnedin \& Hui (1996) and Hui et al (1997) has elucidated the relation between column density peaks ("absorption lines") and the statistics of density peaks, and it has given analytical expressions for the dependence of the shape of the $\operatorname{CDDF} f(N)$ on cosmological parameters. The slope of $f(N)$ was found largely to be determined by the normalization and the slope of the initial mass power spectrum, with changes in the equation of state $T=T(\rho)$ having an additional but smaller impact. The overall normalization of $f(N)$ is given by $\left(\Omega_{b} h^{2}\right)^{2} / \Gamma$; a change in this quantity shifts $f(N)$ horizontally to larger $N$.

Croft et al (1998) have suggested a technique for recovering the initial power spectrum of density fluctuations directly from the fluctuations of the optical depth. The distribution function of pixel fluxes in a Ly $\alpha$ forest spectrum on larger scales is assumed to have originated via gravitational collapse from an initially Gaussian probability distribution of overdensities $\delta$. If true, then the flux probability function can be mapped monotonically, according to the rank of the flux values, back onto a Gaussian probability function for the initial $\delta$. The density power spectrum (width of the Gaussian) $P(k)$ is then known up to a normalization, which can be derived from iterative cosmological simulations with the same $P(k)$ but different normalization until the right flux power spectrum 
is obtained from simulated spectra. The flux power spectrum is unique up to a normalization that can be fixed by observing the mean absorption $\bar{D}$.

POTENTIAL PROBLEMS-UNSOLVED QUESTIONS The new paradigm for the Ly $\alpha$ forest has considerable explanatory power, but that does not mean that it is correct. The interpretation of the absorption systems and the cosmological measurements planned or already performed to date depend on gravitational collapse as the dominant source of structure in the IGM. Even if the hierarchical models are basically correct, it is conceivable that local physical effects may upset some of the cosmological conclusions. A fluctuating radiation field may be a source of nongravitational structure in the forest, as may stellar feedback. How much of the absorption is caused by gas blown out by supernova explosions or stellar winds, and how robust are the cosmological conclusions in that case? Metal enrichment has been found to be common for absorption systems with $\mathrm{H}$ I column densities as low as $\log N(\mathrm{H} \mathrm{I}) \sim 14$ (Cowie et al 1995, Tytler et al 1995). If this is not due to a very early phase of metal enrichment, we have to worry that some process other than gravitational collapse may have formed the metal-enriched $\operatorname{Ly} \alpha$ clouds. The origin of the ionizing radiation and the spectral slope are another source of uncertainty. When and how do reionization and reheating happen, and from where do the photons come? Is collisional reheating important, and how much do stars contribute to the UV flux as a function of time? On the technical side: Do hydrodynamic codes already converge, or how much do the inferred cosmological parameters $\left(\Omega_{b}\right.$, the amount of small-scale structure present) depend on the resolution and size of the simulations, and the numerical technique?

Finally, the cosmological picture itself could be wrong, and the interpretation of the forest as absorption mostly by the IGM (as opposed to distinct galaxies) may be doubtful. Galaxy halos or disks could be big/numerous enough to produce the low column density Ly $\alpha$ forest as well. For low redshift absorption lines, this last possibility has received much attention, and we briefly consider this question next.

\section{THE LYMAN ALPHA FOREST AND GALAXIES}

Is the Ly $\alpha$ forest absorption caused by galaxies? To match the large rate of incidence of typical Ly $\alpha$ absorbers, "normal" (i.e. known types of ) galaxies must possess very large absorption cross sections (Burbidge et al 1977). Alternatively, since the rate of incidence is constraining only the product of number density and geometric cross section of the absorbers, there could be a population of unknown, more numerous objects, subtending a smaller cross section. Two key observations have fuelled the interest in the nature of the 
absorber-galaxy connection. One was the detection of apparently ordinary galaxies at the same (intermediate) redshifts as high column density, Mg II metal absorbers (Bergeron 1986). Subsequent work (Bergeron \& Boissé 1991, Steidel 1995) established an incontrovertible connection between gaseous galactic halos and high column density, metal absorption systems (which we do not discuss here, as it is most relevant for optically thick Lyman limit systems). The other was the widely unanticipated detection of a remnant population of Ly $\alpha$ absorbers in the very local universe $(z \sim 0.003)$ with HST (Bahcall et al 1991, 1993, Morris et al 1991), where the properties of a galaxy can be studied in much greater detail than at high redshift.

\subsection{The Low Redshift Lyman Alpha Forest}

Soon after the discovery of the low $z$ absorbers, galaxy surveys in the fields of the QSOs observed by HST, especially near 3C273, were undertaken to investigate a possible link between absorbers and galaxies. Salzer (1992), Morris et al (1993), and Salpeter \& Hoffman (1995) found redshift coincidences with galaxies for some of the lines, hundreds of kiloparsecs up to megaparsecs away from the absorbers, but there was no unambiguous correspondence between absorbers and individual galaxies. Searches for any low surface brightness objects closer to the LOS to $3 \mathrm{C} 273$, which could have escaped detection, have been performed in H I radio emission (van Gorkom et al 1993), $\mathrm{H} \alpha$ emission (Morris et al 1993, Vogel et al 1995), and in deep broadband optical images (Rauch et al 1996), but all were equally unsuccessful. Morris et al (1993) concluded that there is some correlation between absorbers and galaxies, but the galaxy-absorber cross correlation function is weaker than the galaxy-galaxy correlation. By trying to model the correlation results as a mixture of randomly distributed objects and galactic halos, Mo \& Morris (1994) deduced that galaxy halos constitute only about $25 \%$ of the local absorber population. At first glance, this is at variance with the result of Lanzetta et al (1995), who, based on their large galaxy redshift survey, concluded that most absorption systems are associated with galactic envelopes of typical radius $160 \mathrm{~h}^{-1} \mathrm{kpc}$ (see also Chen et al 1997). Moreover, a significant anticorrelation between the equivalent width and the impact parameter of the LOS from the center of the galaxies was measured. Le Brun et al's (1996) survey showed a weaker anticorrelation, and Bowen et al (1996) saw none at all, although they both confirm Lanzetta et al's results in that there is a region with a high covering factor for absorption around each galaxy, within impact parameters $<200-300 \mathrm{~h}^{-1} \mathrm{kpc}$, dropping rapidly at larger separations. It appears that the conclusions of Morris et al (1991) and Lanzetta et al (1995) can be reconciled if Lanzetta et al's main result, i.e. the existence of well-defined galactic envelopes, is valid only for the relatively strong absorption lines used in their comparison. The weaker lines, 
from which Morris et al (1991) derived a weak galaxy-absorber correlation, could still be caused by a truly intergalactic medium. Absorption systems do exist in voids known from galaxy surveys (Stocke et al 1995, Shull et al 1996), but there appears to be a general trend for the absorbers to trace the same largescale structure as galaxies (Hoffman et al 1995, Stocke et al 1995). In any case, the very large structures causing the stronger common absorption systems in QSO pairs (Bechtold 1994, Dinshaw et al 1994, 1995) cannot be explained by single objects like giant disks or halos: In those cases where several galaxies have been found at the same redshift as the absorber, the typical transverse separation between the galaxies is smaller than the transverse absorber size (Rauch et al 1996). This is reminiscent of Oort's (1981) "uncondensed gas in superclusters." There is amazing redshift agreement (velocity differences $0<\Delta v<20 \mathrm{~km} \mathrm{~s}^{-1}$ ) between the $\mathrm{H}$ I velocity centroids of galaxies and absorption systems with impact parameters as large as $300 \mathrm{~h}^{-1} \mathrm{kpc}$ (van Gorkom et al 1996).

MODELING LOW REDSHIFT LYMAN ALPHA ABSORBERS Attempts at modeling have mainly been concerned with the large cross section required if the low redshift Ly $\alpha$ clouds are parts of the known galaxy population. Maloney (1992) has suggested that the absorption arises in the distant, ionized outer parts of the known population of disk and irregular galaxies. Clouds that are pressure confined by a hotter gas in an extended galactic halo were discussed by Mo (1994). Salpeter (1993) has invoked a new type of extended low redshift disk galaxies, which, in a short burst of star formation, blow out their denser centers and disappear from view while their gas cross section remains (the so-called Cheshire Cat model). The large sizes and low column densities postulated require that these objects must have formed late (Salpeter \& Hoffman 1995). Again, observationally, the large coherence lengths of the absorbers on the sky seem to correspond to groups of galaxies. To get the large covering factor right, these objects must be so close together as to run into each other like circular saws at only slightly higher redshift, which puts us basically back at a continuous IGM. A number of other sources of low $z$ Ly $\alpha$ absorption have been considered, among them tidal tails (Morris \& Van den Berg 1994) and galactic winds (Wang 1995). Galaxy clusters have been seen to cause at least some absorbers (Lanzetta et al 1996), and the strong clustering found among low $z$ absorption lines (Boksenberg 1995, Bahcall et al 1996, Ulmer 1996) hints at the increasing importance of such structures with decreasing redshift.

An open mind is clearly necessary regarding all these possibilities, many of which plausibly may contribute a significant fraction to low $z$ Ly $\alpha$ absorption. How much exactly may be hard to quantify (Sarajedini et al 1996). Again, believers in hierarchical structure formation can expect some consolation from 
simulations. Petitjean et al (1995) predicted that a bimodal distribution of absorbers should be expected, with large galaxy halos with typical radii on the order of $0.5 \mathrm{~h}^{-1} \mathrm{Mpc}$ on one hand and more frequent, lower column density intergalactic absorption occuring in filaments up to several megaparsecs away from the nearest galaxy on the other. Miraculously, this is consistent with all the observational evidence we have.

\subsection{Metal Enrichment in the High Redshift Lyman Alpha Forest}

True to our self-imposed restraints, we deal here only with the low column density forest. For the reasons already mentioned (large transverse sizes, small velocity differences over hundreds of kiloparsecs, probable flattening, absence of voids, weak LOS correlation, low Doppler parameters, and the low column densities themselves), the typical Ly $\alpha$ system at $z \sim 3$ is quite unlikely to be physically associated with a galaxy, other than being part of the gaseous matrix from which galaxies form. One puzzling result remains, however: the finding of widespread metal enrichment in the Ly $\alpha$ forest.

Individual high column density systems with very low metallicities (of order $10^{-3} \odot$ ) exist (e.g. Chaffee et al 1985), but there is no absorption system known with column density above $\log N(\mathrm{H} \mathrm{I})>16$ and a primordial composition. The possibility that there may be a transition at a certain column density from a metal-enriched gas to a primordial gas has led to intensive searches for weak metal lines in low column density absorbers. Norris et al (1983), Williger et al (1989), and Lu (1991) used a "shift and stack" method to search for various metals in the low column density forest. To maximize the signal-to-noise ratio, the spectra are shifted to the rest frame indicated by each Ly $\alpha$ and added, and the expected positions of metal lines are searched for a signal. A tentative detection of C IV, corresponding to a carbon depletion relative to solar, of $[\mathrm{C} / \mathrm{H}] \approx-3.1$, was made by Lu et al (1991). Meyer \& York (1987) pointed out that data with an increasing signal-to-noise ratio show increasing numbers of individual weak C IV systems. The subject attracted renewed interest when Keck spectra showed that most Ly $\alpha$ systems with $\mathrm{H}$ I column densities $10^{15}$ and roughly half of all Ly $\alpha$ systems with column densities $>3 \times 10^{14} \mathrm{~cm}^{-2}$ have associated C IV lines, corresponding to a typical metallicity of $Z=10^{-2} Z_{\odot}$ (Cowie et al 1995, Tytler et al 1995, Songaila \& Cowie 1996). Unfortunately, the detection threshold for C IV is close enough to make it hard to determine whether the decreasing rate of detections is a genuine turnover to primordial composition below a few times $10^{14}$ or just a selection effect. It is interesting that in the CDM models, a column density contour of $10^{14}$ at redshift 3 delineates the transition between a continuous filamentary structure in the universe, with typical widths of less than $100 \mathrm{kpc}$, and the voids. Galaxies would have to spill metals only 
within the filaments to create the widespread metal enrichment observed, and they could still have left most of the volume of the universe pristine. Thus a drop in metallicity at a few times $10^{14}$ would not come unexpectedly. A relatively uniform metal abundance across the whole column density range could be an indication of an earlier phase of nucleosynthesis.

\section{THE HELIUM LYMAN ALPHA FOREST}

Observations of absorption by the He I and He II Lyman series provide another independent source of information on the state of the IGM and the UV background radiation (Sargent et al 1980). When comparing the Doppler parameters of the He II $304-\AA \AA$ and H I 1215 - $\AA$ lines, it is possible in principle to use the difference in atomic masses to measure the contributions from bulk and thermal motions to the line broadening separately, so theories of the kinematics of the cosmic gas can be tested. The far-UV He II Lyman edge at $228 \AA$ probes the intensity of the UV background at much shorter wavelengths than the $\mathrm{H} \mathrm{I}$ edge. As the photoionization rates are dominated by the intensity of the ionizing radiation near the respective ionization edges, then in principle, measurements of the He II and H I column densities can fix the spectral shape of the UV background in the vicinity of two points, $228 \AA$ and $912 \AA$. This comparison can be done as a function of wavelength (or redshift), so it is possible to measure the spatial spectral fluctuations of the UV background caused by local UV sources and by fluctuations in the intergalactic absorption and, perhaps at higher redshifts, to study the progress of reionization (e.g. Donahue \& Shull 1987, Shapiro \& Giroux 1987, Miralda-Escudé \& Rees 1993, Madau \& Meiksin 1994, Shapiro et al 1994, Giroux et al 1995).

In a photoionized optically thin gas, He III is the dominant ionization state; the remaining $\mathrm{He}$ is expected to be mostly in the form of singly ionized $\mathrm{He}$ II. For realistic spectral intensity distributions (except for the very soft UV background caused by decaying neutrinos; Sciama 1990), He I is undetectable in the optically thin clouds of the Ly $\alpha$ forest proper, although it should be present in Lyman limit systems (Miralda-Escudé \& Ostriker 1992). Indeed, He I yielded the first detection of helium at high redshift, when absorption by its 504-Å line was found in HST FOS data of several optically thick $(z \sim 2)$ metal systems towards the bright QSO HS1700+64 (Reimers et al 1992, Reimers \& Vogel 1993). The ionization state of He I is less easy to interpret because of the possible presence of internal sources of radiation and unknown shielding effects.

\subsection{The He II Lyman Alpha Forest}

Because of its relative strength, He II $304 \AA$ is likely to be a better tracer of the low density baryon distribution than even H I Ly $\alpha$. The principal observable 
of the He II Ly $\alpha$ forest is the ratio of the GP optical depths of He II to H I:

$$
\frac{\tau_{\mathrm{He} \text { II }}}{\tau_{\mathrm{H} \mathrm{I}}}=\frac{1}{4} \frac{N_{\mathrm{He} \mathrm{II}}}{N_{\mathrm{H} \mathrm{I}}}=\frac{1}{4} \eta=0.43 \frac{J_{\mathrm{H} \mathrm{I}}}{J_{\mathrm{He} \mathrm{II}}},
$$

where $N_{\mathrm{He} \mathrm{II}}$ and $N_{\mathrm{H} \mathrm{I}}$ are the column densities of He II and H I, respectively (Miralda-Escudé 1993). The last equation is valid for an optically thin gas, where the ionization equilibrium is governed by photoionization and both $\mathrm{H}$ and $\mathrm{He}$ are highly ionized. $J_{\mathrm{He} \mathrm{II}}$ and $J_{\mathrm{HI}}$ are the intensities of the ionizing radiation field weighted with the frequency dependence of the photoionization cross sections. Thus the relative strength of the GP troughs are only dependent on the ratios of the ionizing fluxes, a situation that can be cast in terms of the column density ratio $\eta$ or the ratio of the intensities at the absorption edges, the softness parameter $S_{L}$ (Madau \& Meiksin 1994),

$$
\eta=\frac{N_{\mathrm{He} \mathrm{II}}}{N_{\mathrm{H} \mathrm{I}}} \approx 1.8 \frac{J_{912}}{J_{228}}=1.8 S_{L} .
$$

The He II/H I ratio $\eta$ can range from values of a few to a thousand, depending on the spectral slope of the ionizing radiation. The shape of the ionizing spectrum depends on the relative mix between "hard" (AGNs) and "soft" (stellar) sources and on the details of radiative transfer by the IGM (Bechtold et al 1987, Shapiro \& Giroux 1987, Miralda-Escudé \& Ostriker 1990, Meiksin \& Madau 1993, Giroux \& Shapiro 1996, Haardt \& Madau 1996).

Thus a He II 304- $\AA$ GP trough should appear more prominent than the corresponding H I trough (Miralda-Escudé 1993), allowing for a more sensitive measurement of the distribution of gas in low density regions. For the actual He II forest of discrete absorption lines, a relation similar to Equation 19 holds, where $\tau$ is now replaced by $\tau_{\text {eff }}$ as defined earlier (Miralda-Escudé 1993):

$$
\frac{\tau_{\text {eff He II }}}{\tau_{\text {eff H I }}}=\left(0.43 \frac{J_{\mathrm{H} \mathrm{I}}}{J_{\text {He II }}}\right)^{\beta-1}\left(\frac{b_{\text {He II }}}{b_{\text {H I }}}\right)^{2-\beta} \text {. }
$$

This relation gives the optical depths for a $\operatorname{Ly} \alpha$ forest of individual lines above a certain optical depth threshold, assuming that the column density distribution of H I Ly $\alpha$ lines is a power law with index $\beta$. The Doppler parameters are $b_{\mathrm{He} \text { II }}$ and $b_{\mathrm{H} \mathrm{I}}$. Clearly, together with the strong He II GP effect, there should be a He II forest stronger than the corresponding $\mathrm{H} \mathrm{I} \mathrm{Ly} \alpha$ forest by a similar factor.

OBSERVATIONS Only a small fraction of all QSOs are suitable for a search for He II absorption. The short wavelength of $304 \AA$ requires an object to be redshifted to at least $z>2-3$ for the He II line to enter the far-UV bands accessible to the Hubble Space Telescope (HST) or the Hopkins Ultraviolet Telescope (HUT). The QSO has to be bright enough for a spectrum to be taken, 
and most importantly, there must be flux down to the wavelength range of interest. The average blanketing of the spectrum by Ly $\alpha$ lines (the "Lyman valley" Møller \& Jakobsen 1990) and especially the total blackout imposed by individual intervening Lyman limit systems below $912 \AA$ in their rest frame renders the large majority of QSOs useless for a He II search (Picard \& Jakobsen 1993), and surveys of known QSOs for residual UV flux (e.g. Jakobsen et al 1993) have to be mounted to select suitable candidates.

To date, there are five detections of the He II forest. A He II absorption "break" blueward of the He II emission line was first seen with the HST FOC far-UV prism by Jakobsen et al (1994) in the LOS to Q0302-003 $\left(z_{e m}=\right.$ 3.29 ), leading to an estimate for the mean optical depth, $\tau_{\mathrm{He}} \mathrm{II}=3.2_{-1.1}^{+\infty}$. The object was reobserved at higher resolution with the GHRS instrument by Hogan et al $(1997)\left(\tau_{\mathrm{He} \mathrm{II}} \approx 2\right.$, beyond the proximity effect region). Tytler et al (1995) obtained $\tau_{\mathrm{He} \mathrm{II}}=1.0 \pm 0.2$, later corrected to $\tau_{\mathrm{He} \mathrm{II}}>1.5$ (D Tytler $\&$ P Jakobsen, unpublished data) in the LOS to Q1937-69 $\left(z_{e m}=3.18\right)$. Davidsen et al (1996) observed the object HS1700+6416 with HUT to obtain $\tau_{\mathrm{He} \mathrm{II}}=$ $1.0 \pm 0.2$, at $\langle z\rangle=2.4$. Reimers et al (1997), in the LOS to HE2347-4342 $\left(z_{e m}=2.89\right)$, found the He II absorption to consist of patches with a high continuous GP component, $\tau_{\mathrm{He} \text { II }}=4.8_{-2}^{+\infty}$, in addition to the contribution expected from the discrete lines, which alternate with regions with less GP absorption, $\tau_{\mathrm{He} \mathrm{II}} \approx 3$.

INTERPRETATION Given the large uncertainties, all $\tau$ measurements to date seem to be consistent with each other, if (the expected) increase in the optical depth with redshift is taken into account. Constraining the strength and shape of the ionizing radiation from the absorbed flux requires a knowledge of the clumpiness of the gas because of the exponential dependence of the absorbed flux on the optical depth. According to Equation 20, the relative strengths of the absorption by $\mathrm{H} \mathrm{I}$ and $\mathrm{He}$ II depend on the amount of bulk motion relative to thermal motion, the CDDF (slope and possible cutoff at low column density), and the relative contribution from a diffuse absorption trough. Arguments have been put forward both against (Songaila et al 1995) and in favor (Hogan et al 1997, Reimers et al 1997, Zheng et al 1998) of the existence of such a trough in addition to the line absorption expected from translating the known H I Ly $\alpha$ forest into a He II forest. If the proponents of additional trough absorption are correct, this may also imply that the H I CDDF has been overcorrected for confusion and does not extend to as low a column density as previously assumed. Superficially, this argument sounds like the return of the lines-versustrough debate familiar from the H I GP effect, but there is new information to be gained by studying the detailed structure of the low density He II absorption. The larger optical depth of He II highlights very low density structure in voids, 
which may be too weak to be constrained usefully with optical H I Ly $\alpha$ forest spectra. Eventually such observations will constrain the spatial fluctuations of the ionizing radiation field and the density field in a large fraction of the volume of the universe. The question of whether the spatial variations of the diffuse absorption can be (or should be) parametrized, as "lines" may have to await the arrival of better data. In any case, the finding of a substantial amount of He II absorption from voids is an important consistency check for hierarchical structure formation models (Zhang et al 1995, 1997, Croft et al 1997).

At the time of this writing, the spectral shape of the radiation field still is not well constrained (Sethi \& Nath 1997, Reimers et al 1997, Zheng et al 1998). An interesting twist has been added by the detection of patchy He II absorption, which is inconsistent with a uniform radiation field. Reimers et al (1997) invoked incomplete reionization of He II as a possible explanation, an effect predicted to produce saturated absorption troughs (Meiksin \& Madau 1993). However, it is hard to tell how strongly saturated the troughs really are. The observations still may be consistent with a fully reionized He II if the troughs are caused by local fluctuations in the He II ionizing background (Miralda-Escudé 1998).

\section{PROSPECTS}

Although most of the observational basis and many theoretical aspects of Ly $\alpha$ forest absorbers had already been established over the past two decades, high signal-to-noise spectroscopy of QSOs with large telescopes, the extension of the wavelength range into the far UV with satellites, and the success of hydrodynamic cosmological simulations have begun to turn the study of the Ly $\alpha$ forest from a somewhat esoteric appendix of cosmology into a cosmologically useful tool. If our current understanding is correct, the high redshift Ly $\alpha$ forest absorption is the observational signature of most of the baryons throughout most of the history of the universe. Perhaps most important, we are looking at the typical fate of matter, without any reference to luminous objects.

The new general picture of the forest that has emerged is still in need of more secure observational conformation before we can trust any quantitative cosmological conclusions. The simulations agree with some aspects of the data, but are they unique and consistent with each other? More detailed comparisons with the data are called for, partly with new techniques of data analysis tailored to maximize the discriminative power. Semianalytical work still has an important function in guiding our understanding of the actual physics and in exploring parameter space quickly. The observers need to explore the limitations of the observational techniques and systematic effects in the data, formerly gracefully 
hidden by a veil of noise but now exposed by large mirrors to the strict eye of numerical theory.

We can expect new observational facilities to enlarge the scope of absorption line studies considerably. The projected Cosmic Origins Spectrograph (COS) will increase the spectroscopic efficiency of the HST, benefiting almost all of the observational areas mentioned here. The Sloan Digital Sky Survey (SDSS) should produce large numbers of QSOs useful for spectroscopic follow-up, to study (for example) the large-scale structure from intergalactic absorption in three dimensions. Even low resolution QSO spectra will be useful for cosmological purposes. And the use of large optical telescopes is inevitable for studies of gravitationally lensed QSOs, the absorber-galaxy connection at any redshift, and of course, for most projects involving narrow metal absorption lines, a subject of increasing relevance for our understanding of the process of galaxy formation.

Unfortunately, other applications of absorption lines, like the remarkably militant debate about the deuterium to hydrogen ratio, the whole topic of intervening metal absorption systems and its relevance for galaxy formation, and the process of reionization, could not be treated here. Perhaps we have ignored them with some justification; these subjects are currently in such a state of flux that any review could be out of date before going to press.

\section{ACKNOWLEDGMENTS}

I am grateful to Bob Carswell, Martin Haehnelt, Jordi Miralda-Escudé, and Wal Sargent for reading earlier drafts and to NASA for support through grant HF01075.01-94A from the Space Telescope Science Institute, which is operated by the Association of Universities for Research in Astronomy, Inc., under NASA contract NAS5-26555.

Visit the Annual Reviews home page at

http://www.AnnualReviews.org.

\section{Literature Cited}

Acharya M, Khare P. 1993. J. Astrophys. Astron. 14:97

Arons J. 1972. Ap. J. 172:553

Arons J, McCray R. 1969. Astrophys. Lett. $5: 123$

Atwood B, Baldwin RA, Carswell RF. 1985. Ap. J. 292:58

Babul A. 1990. Ap. J. 349:429

Babul A. 1991. MNRAS 248:177

Babul A, Rees MJ. 1992. MNRAS 255:346

Bahcall JN, Bergeron J, Boksenberg A, Hartig GF, Jannuzi BT. 1996. Ap. J. 457:19
Bahcall JN, Jannuzi BT, Schneider DP, Hartig GF, Bohlin R, Junkkarinen V. 1991. Ap. J. Lett. 377:5

Bahcall JN, Peebles PJE. 1969. Ap. J. 156:L7

Bahcall JN, Salpeter EE. 1965. Ap. J. 142:1677

Bahcall JN, Spitzer L. 1969. Ap. J. 156:L63

Bajtlik S, Duncan RC, Ostriker JP. 1988. Ap. J. 327:570

Baldwin JA, Burbidge EM, Burbidge GR, Hazard C, Robinson LB, et al. 1974. Ap. J. 193:513

Barcons X, Fabian AC. 1987. MNRAS 224:675 
Barcons X, Fabian AC, Rees MJ. 1991. Nature 350:685

Barcons X, Webb JK. 1990. MNRAS 244:30p

Barcons X, Webb JK. 1991. MNRAS 253:207

Baron E, Carswell RF, Hogan CJ, Weymann RJ. 1989. Ap. J. 337:609

Bechtold J. 1987. In High Redshift and Primeval Galaxies, ed. J Bergeron, D Kunth, B RoccaVolmerange, J Tran Thanh Van. Gif-surYvette: Ed. Front.

Bechtold J. 1994. Ap. J. Suppl. 91:1

Bechtold J. 1995. In QSO Absorption Lines, ed. G Meylan, p. 299. Berlin: Springer-Verlag

Bechtold J, Crotts APS, Duncan RC, Fang Y. 1994. Ap. J. 437:83

Bechtold J, Green RF, Weymann RJ, Schmidt M, Estabrook F, et al. 1984. Ap. J. 281:76

Bechtold J, Weymann RJ, Zuo L, Malkan MA. 1987. Ap. J. 315:118

Bergeron J. 1986. Astron. Astrophys. 155:L8

Bergeron J, Boissé P. 1991. Astron. Astrophys. 243:344

Bi H. 1993. Ap. J. 405:479

Bi H, Börner G, Chu Y. 1989. Astron. Astrophys. 218:29

Bi H, Börner G, Chu Y. 1991. Astron. Astrophys. 247:276

Bi H, Börner G, Chu Y. 1992. Astron. Astrophys. 266:1

Bi H, Davidsen AF. 1997. Ap. J. 479:523

Black J. 1981. MNRAS 197:553

Boksenberg A. 1995. In QSO Absorption Lines, ed. G Meylan, p. 253. Berlin: SpringerVerlag

Bond JR, Kofman L, Pogosyan D. 1996. Nature 380:603

Bond JR, Szalay AS, Silk J. 1988. Ap. J. 324:627

Bond JR, Wadsley JW. 1997. Computational Astrophys. Proc. 12th Kingston Conf., Halifax, October 1996, ed. D Clarke, M West, ASP Conf. Ser., p. 323. San Francisco: Astron. Soc. Pac.

Bowen DV, Blades J, Pettini M. 1996. Ap. J. 464:141

Burbidge EM, Lynds CR, Burbidge GR. 1966. Ap. J. 144:447

Burbidge G, O'Dell SL, Roberts DH, Smith HE. 1977. Ap. J. 218:33

Carswell RF. 1988. QSO Absorption Lines: Probing the Universe, Proc. QSO Absorption Line Meeting, Baltimore 1987, ed. JC Blades, D Turnshek, CA Norman, p. 91. Cambridge: Cambridge Univ. Press

Carswell RF, Lanzetta KM, Parnell HC, Webb JK. 1991. Ap. J. 371:36

Carswell RF, Morton DC, Smith MG, Stockton AN, Turnshek DA, Weymann RJ. 1984. Ap. J. 278:486

Carswell RF, Rees MJ. 1987. MNRAS 224:13

Carswell RF, Webb JK, Baldwin JA, Atwood B. 1987. Ap. J. 319:709
Carswell RF, Whelan JAJ, Smith MG, Boksenberg A, Tytler D. 1982. MNRAS 198:91

Cen R, Miralda-Escudé J, Ostriker JP, Rauch M. 1994. Ap. J. Lett. 437:9

Cen R, Ostriker JP. 1993. Ap. J. 417:404

Cen R, Simcoe RA. 1997. Ap. J. 483:8

Chaffee FH, Foltz CB, Weymann RJ, Röser H-J, Latham DW. 1985. Ap. J. 292:362

Chaffee FH, Weymann RJ, Strittmatter PA, Latham DW. 1983. Ap. J. 252:10

Charlton JC, Anninos P, Zhang Y, Norman M. 1997. Ap. J. 485:26

Charlton JC, Salpeter EE, Hogan CJ. 1993. Ap. J. 402:493

Charlton JC, Salpeter EE, Linder SM. 1994. Ap. J. 430:29

Chen H-W, Lanzetta KM, Webb JK, Barcons X. 1998. Ap. J. In press

Chernomordik VV. 1988. Sov. Astron. 32:6

Chernomordik VV. 1994. Ap. J. 440:431

Chernomordik VV, Ozernoy LM. 1983. Nature 303:153

Chernomordik VV, Ozernoy LM. 1993. Ap. J. 404:5

Cooke AJ, Espey B, Carswell RF. 1997. MNRAS 284:552

Cowie LL, Songaila A, Kim T-S, Hu EM. 1995. Astron. J. 109:1522

Cristiani S, D'Odorico S, D'Odorico V, Fontana A, Giallongo E, Savaglio S. 1997. MNRAS 285:209

Cristiani S, D’Odorico S, Fontana A, Giallongo E, Savaglio S. 1995. MNRAS 273:1016

Croft RA, Weinberg DH, Katz N, Hernquist L. 1997. Ap. J. 488:532

Croft RA, Weinberg DH, Katz N, Hernquist L. 1998. Ap. J. 495:44

Crotts APS. 1987. MNRAS 228:41

Crotts APS. 1989. Ap. J. 336:550

Davé R, Hernquist L, Weinberg DH, Katz N. 1997. Ap. J. 477:21

Davidsen AF, Kriss GA, Zheng W. 1996. Nature 380:47

Dinshaw N, Foltz CB, Impey CD, Weymann RJ, Morris SL. 1995. Nature 373:223

Dinshaw N, Impey CD, Foltz CB, Weymann RJ, Chaffee FH. 1994. Ap. J. 437:87

Dobrzycki A, Bechtold J. 1991. Ap. J. Lett. 377:69

Donahue M, Shull JM. 1987. Ap. J. 323:L13

Donahue M, Shull JM. 1991. Ap. J. 383:511

Doroshkevich AG, Muecket JP. 1985. Sov. Astron. Lett. 11:331

Doroshkevich AG, Shandarin SF. 1977. MNRAS 179:95

Duncan RC, Ostriker JP, Bajtlik S. 1989. Ap. J. 345:39

Duncan RC, Vishniac ET, Ostriker JP. 1991. Ap. J. 368:1

Espey BR. 1993. Ap. J. 411:59

Fang LZ. 1991. Astron. Astrophys. 244:1 
Fang Y, Duncan RC, Crotts APS, Bechtold J. 1996. Ap. J. 462:77

Fardal MA, Shull JM. 1993. Ap. J. 415:524

Fernandez-Soto A, Barcons X, Carballo R, Webb JK. 1995. MNRAS 277:235

Fernandez-Soto A, Lanzetta KM, Barcons X, Carswell RF, Webb JK, Yahil A. 1996. Ap. J. 460:85

Foltz CB, Weymann RJ, Röser HJ, Chaffee FH. 1984. Ap. J. 281:1

Francis PJ, Hewett PC. 1993. Astron. J. 105:1633

Fransson C, Epstein R. 1982. Astron. Astrophys. 198:1127

Giallongo E. 1991. MNRAS 251:541

Giallongo E, Cristiani S. 1990. MNRAS 247:696

Giallongo E, Cristiani S, D'Odorico S, Fontana A, Savaglio S. 1996. Ap. J. 466:46

Giallongo E, Cristiani S, Fontana A, Trèvese D. 1993. Ap. J. 416:137

Giallongo E, Cristiani S, Trèvese D. 1992. Ap. J. 398:9

Giallongo E, D'Odorico S, Fontana A, McMahon R, Savaglio S, et al. 1994. Ap. J. Lett. 425:1

Giallongo E, Petitjean P. 1994. Ap. J. 426:61

Giroux ML, Fardal MA, Shull JM. 1995. Ap. J. 451:477

Giroux ML, Shapiro P. 1996. Ap. J. Suppl. 102:191

Gnedin NY, Hui L. 1996. Ap. J. Lett. 472:73

Gnedin NY, Hui L. 1998. MNRAS. In press

Goldreich P, Sargent WLW. 1976. Comments Astrophys. 6:133

Gunn JE, Peterson BA. 1965. Ap. J. 142:1633

Haardt F, Madau P. 1996. Ap. J. 461:20

Haehnelt M, Rauch M, Steinmetz M. 1996a. MNRAS 283:1055

Haehnelt M, Steinmetz M. 1998. MNRAS. In press

Haehnelt M, Steinmetz M, Rauch M. 1996b. Ap. J. 465:95

Hernquist L, Katz N, Weinberg D, MiraldaEscudé J. 1996. Ap. J. Lett. 457:51

Hoffman GL, Lewis BM, Salpeter EE. 1995. Ap. J. 441:28

Hogan CJ, Anderson SF, Rugers MH. 1997. Astron. J. 113:1495

Hu EM, Kim T-S, Cowie LL, Songaila A, Rauch M. 1995. Astron. J. 110:1526

Hui L, Gnedin NY. 1997. MNRAS 292:27

Hui L, Gnedin NY, Zhang Y. 1997. Ap. J. 486:599

Hunstead RW, Murdoch HS, Pettini M, Blades JC. 1988. Ap. J. 329:527

Hunstead RW, Pettini M. 1991. In Proc. ESO Mini-Workshop QSO Absorption Lines, ed. PA Shaver, EJ Wampler, AM Wolfe, ESO Sci. Rep. No. 9, p. 11. Garching: Eur. South. Obs.

Hunstead RW, Pettini M, Blades JC, Murdoch
HS. 1986. Proc. 124th IAU Symp., Beijing, ed. A Hewitt, G Burbidge, Z-F Fang. Dordrecht: Reidel

Ikeuchi S. 1986. Astrophys. Space Sci. 118: 509

Ikeuchi S, Murakami I, Rees MJ. 1988. MNRAS 236:21p

Ikeuchi S, Ostriker JP. 1986. Ap. J. 301:522

Impey CD, Petry CE, Malkan MA, Webb W. 1996. Ap. J. 463:473

Jakobsen P, Albrecht R, Barbieri C, Blades JC, Boksenberg A, et al. 1993. Ap. J. 417:528

Jakobsen P, Boksenberg A, Deharveng JM, Greenfield P, Jedrzejewski R, Paresce F. 1994. Nature 370:35

Jenkins EB, Ostriker JP. 1991. Ap. J. 376:33

Katz N, Weinberg DH, Herquist L, MiraldaEscudé J. 1996. Ap. J. Lett. 457:57

Kim T-S, Hu EM, Cowie LL, Songaila A. 1997. Astron. J. 114:1

Kinman TD. 1966. Ap. J. 144:1232

Kirkman D, Tytler D. 1997. Ap. J. 484:672

Kovner I, Rees MJ. 1989. Ap. J. 345:52

Kulkarni VP, Fall SM. 1993. Ap. J. 413:63

Kulkarni VP, Huang K, Green RF, Bechtold J, Welty DE, York DG. 1996. MNRAS 279:197

Lanzetta KM. 1988. Ap. J. 332:96

Lanzetta KM, Bowen DB, Tytler D, Webb JK. 1995. Ap. J. 442:538

Lanzetta KM, Webb JK, Barcons X. 1996. Ap. J. Lett. 456:17

Le Brun V, Bergeron J, Boissé P. 1996. Astron. Astrophys. 306:691

Liu XD, Jones BJT. 1988. MNRAS 230:481

Liu XD, Jones BJT. 1990. MNRAS 242:678

Loeb A, Eisenstein DJ. 1995. Ap. J. 448:17

Lu L. 1991. Ap. J. 379:99

Lu L, Sargent WLW, Womble DS, Takada-Hidai M. 1996. Ap. J. 472:509

Lu L, Wolfe AM, Turnshek DA. 1991. Ap. J. 367:19

Lu L, Zuo L. 1994. Ap. J. 426:502

Lynds CR. 1970. IAU Symp. 44:127

Lynds CR, Stockton AN. 1966. Ap. J. 144:446

Madau P, Meiksin A. 1991. Ap. J. 374:6

Madau P, Meiksin A. 1994. Ap. J. 433:L53

Maloney P. 1992. Ap. J. 398:89

McGill C. 1990. MNRAS 242:544

Meiksin A. 1994. Ap. J. 431:109

Meiksin A, Bouchet FR. 1995. Ap. J. Lett. 448:85

Meiksin A, Madau P. 1993. Ap. J. 412:34

Melott A. 1980. Ap. J. 268:630

Meyer DM, York DG. 1987. Ap. J. 315:5

Milgrom M. 1988. Astron. Astrophys. 202:9

Miralda-Escudé J. 1993. MNRAS 262:273

Miralda-Escudé J. 1998. Ap. J. Submitted

Miralda-Escudé J, Cen R, Ostriker JP, Rauch M. 1996. Ap. J. 471:582

Miralda-Escudé J, Ostriker JP. 1990. Ap. J. 350:1 
Miralda-Escudé J, Ostriker JP. 1992. Ap. J. 392:15

Miralda-Escudé J, Rees MJ. 1993. MNRAS 260: 617

Miralda-Escudé J, Rees MJ. 1994. MNRAS 266: 343

Mo HJ. 1994. MNRAS 269:49

Mo HJ, Miralda-Escudé J. 1996. Ap. J. 469:589

Mo HJ, Miralda-Escudé J, Rees MJ. 1993. MNRAS 264:705

Mo HJ, Morris SL. 1994. MNRAS 269:52

Mo HJ, Xia XY, Deng ZG, Börner G, Fang L-Z. 1992. Astron. Astrophys. 256:L23

Møller P, Jakobsen P. 1990. Astron. Astrophys. 228:299

Møller P, Kjaergaard P. 1991. Astron. Astrophys. 258:234

Morris SL, Van den Berg S. 1994. Ap. J. 427:696

Morris SL, Weymann RJ, Dressler A, McCarthy PJ, Smith BA, et al. 1993. Ap. J. 419:524

Morris SL, Weymann RJ, Savage BD, Gilliland RL. 1991. Ap. J. Lett. 377:21

Muecket JP, Mueller V. 1987. Ap. Space Sci. 139:163

Muecket JP, Petitjean P, Kates RE, Riediger R. 1996. Astron. Astrophys. 308:17

Murakami I, Ikeuchi S. 1990. PASJ 42:L11

Murakami I, Ikeuchi S. 1993. Ap. J. 409:42

Murdoch HS, Hunstead RW, Pettini M, Blades JC. 1986. Ap. J. 309:19

Norris J, Hartwick FDA, Peterson BA. 1983. MNRAS 273:450

O’Brian PT, Gondhalekar PM, Wilson R. 1988. MNRAS 233:801

Oke JB, Korycansky DG. 1982. Ap. J. 255:11

Oort HJ. 1981. Astron. Astrophys. 94:359

Ostriker JP, Bajtlik S, Duncan RC. 1988. Ap. J. 327:350

Ostriker JP, Cowie LL. 1981. Ap. J. 243:127

Ostriker JP, Ikeuchi. 1983. Ap. J. Lett. 268:63

Ozernoy LM, Chernomordik VV. 1976. Sov. Astron. Lett. 2:375

Pando J, Fang L-Z. 1996. Ap. J. 459:1

Paresce F, McKee CF, Bowyer S. 1980. Ap. J. 240:389

Parnell HC, Carswell RF. 1988. MNRAS 230: 491

Peacock J. 1991. Nature 349:190

Peterson BA. 1978. In The Large Scale Structure of the Universe, ed. MS Longair, J Einasto, p. 389. Dordrecht: Reidel

Petitjean P, Bergeron J, Carswell RF, Puget JL. 1993a. MNRAS 260:67

Petitjean P, Muecket JP, Kates RE. 1995. Astron. Astrophys. 295:L12

Petitjean P, Rauch M, Carswell RF. 1994. Astron. Astrophys. 291:29p

Petitjean P, Webb JK, Rauch M, Carswell RF, Lanzetta KM. 1993b. MNRAS 262:499

Pettini M, Hunstead RW, Smith LJ, Mar DP. 1990. MNRAS 246:545
Picard A, Jakobsen P. 1993. Astron. Astrophys. 276:331

Pierre M, Shaver PA, Iovino A. 1988. Astron. Astrophys. 197:3

Press WH, Rybicki GB, Schneider DP. 1993. Ap. J. 414:64

Prochaska JX, Wolfe AM. 1997. Ap. J. 487:73

Rauch M. 1996. In Cold Gas at High Redshift, ed. MN Bremer, et al, p. 137. Dordrecht: Kluwer

Rauch M, Carswell RF, Chaffee FH, Foltz CB, Webb JK, et al. 1992. Ap. J. 390:387

Rauch M, Carswell RF, Webb JK, Weymann RJ. 1993. MNRAS 260:589

Rauch M, Haehnelt MG. 1995. MNRAS 275:76

Rauch M, Miralda-Escudé J, Sargent WLW, Barlow TA, Weinberg DH, et al. 1997. Ap. J. 489:7

Rauch M, Weymann RJ, Morris SL. 1996. Ap. J. 458:518

Rees MJ. 1986. MNRAS 218:25

Rees MJ. 1988. QSO Absorption Lines: Probing the Universe, Proc. QSO Absorption Line Meeting, Baltimore 1987, ed. JC Blades, D Turnshek, CA Norman, p. 107. Cambridge: Cambridge Univ. Press

Rees MJ, Setti G. 1970. Astron. Astrophys. 8:410

Reimers D, Köhler S, Wisotzki L, Groote D, Rodriguez-Pascal P, Wamsteker W. 1997. Astron. Astrophys. 327:890

Reimers D, Vogel S. 1993. Astron. Astrophys. 276:L13

Reimers D, Vogel S, Hagen HJ, Engels D, Groote D, et al. 1992. Nature 360:561

Reisenegger A, Miralda-Escudé J. 1995. Ap. J. 449:476

Riediger R, Petitjean P, Muecket JP. 1998. Astron. Astrophys. 329:30

Salpeter EE. 1993. Astron. J. 106:1265

Salpeter EE, Hoffman GL. 1995. Ap. J. 441:51

Salzer JJ. 1992. Astron. J. 103:385

Sarajedini V, Green RF, Jannuzi BT. 1996. Ap. J. 457:542

Sargent WLW, Steidel CC, Boksenberg A. 1989. Ap. J. 69:703

Sargent WLW, Young PJ, Boksenberg A, Carswell RF, Whelan JAJ. 1979. Ap. J. 230:49

Sargent WLW, Young PJ, Boksenberg A, Tytler D. 1980. Ap. J. Suppl. 42:41

Sargent WLW, Young PJ, Schneider DP. 1982. Ap. J. 256:374

Savaglio S, Cristiani S, D'Odorico S, Fontana A, Giallongo E, Molaro P. 1997. Astron. Astrophys. 318:347

Scheuer PAG. 1965. Nature 207:963

Schneider D, Schmidt M, Gunn JE. 1991. Astron. J. 102:837

Schwarz J, Ostriker JP, Yahil A. 1975. Ap. J. 202:1

Sciama D. 1990. Ap. J. 364:549 
Sethi SK, Nath BB. 1997. MNRAS 289:634

Shapiro PR, Giroux ML. 1987. Ap. J. 321:L107

Shapiro PR, Giroux ML, Babul A. 1994. Ap. J. 427:25

Shaver PA, Robertson JG. 1983. Ap. J. 268:57

Shklovski IS. 1965. Sov. Astron. 8:638

Shull JM, Stocke JT, Penton S. 1996. Astron. J. 111:72

Smette A. 1995. In QSO Absorption Lines, ed. G Meylan, p. 275. Berlin: Springer-Verlag

Smette A, Robertson JG, Shaver PA, Reimers D, Wisotzki L, Koehler T. 1995. Astron. Astrophys. 113:199

Smette A, Surdej J, Shaver PA, Foltz CB, Chaffee FH, et al. 1992. Ap. J. 389:39

Songaila A, Cowie LL. 1996. Astron. J. 112:335

Songaila A, Hu EM, Cowie LL. 1995. Nature 375:124

Spitzer L. 1956. Ap. J. 124:20

Sramek RA, Weedman DW. 1978. Ap. J. 221:468

Steidel CC. 1995. In QSO Absorption Lines, ed. G Meylan, p. 139. Berlin: Springer-Verlag

Steidel CC, Sargent WLW. 1987a. Ap. J. 313:171

Steidel CC, Sargent WLW. 1987b. Ap. J. 318:11

Stocke JT, Shull JM, Penton S, Donahue M, Carilli C. 1995. Ap. J. 451:24

Stockton AN, Lynds CR. 1966. Ap. J. 144:451

Trevese D, Giallongo E, Camurani L. 1992. Ap J. 398:491

Tytler D. 1987a. Ap. J. 321:49

Tytler D. 1987b. Ap. J. 321:69

Tytler D, Fan X-M, Burles S, Cottrell L, Davis C, et al. 1995. In QSO Absorption Lines, ed. G Meylan, p. 289. Berlin: Springer-Verlag

Ulmer A. 1996. Ap. J. 473:110

Umemura M, Ikeuchi S. 1985. Ap. J. 299:583

van Gorkom J, Bahcall JN, Jannuzi B, Schneider DP. 1993. Astron. J. 106:2213

van Gorkom J, Carilli CL, Stocke JT, Perlman ES, Shull JM. 1996. Astron. J. 112:1397

Vishniac ET, Bust GS. 1987. Ap. J. 319:14

Vishniac ET, Ostriker JP, Bertschinger E. 1985. Ap. J. 291:399

Vogel SN, Weymann RJ, Rauch M, Hamilton T. 1995. Ap. J. 441:162

Vogt SS, Allen SL, Bigelow BC, Bresee L, Brown B, et al. 1994. SPIE 2198:362

Wadsley JW, Bond JR. 1997. In Computational Astrophys. Proc. 12th Kingston Conf. Halifax, October 1996, ed. D Clarke, M West, ASP Conf. Ser., p. 332. San Francisco: Astron. Soc. Pac.
Wagoner R. 1967. Ap. J. 149:465

Walsh D, Carswell RF, Weymann RJ. 1979. Nature 179:381

Wang B. 1995. Ap. J. 444:17

Webb JK. 1986. Proc. 124th IAU Symp. Bejing, ed. A Hewitt, G Burbidge, Z-F Fang, p. 803. Dordrecht: Reidel

Webb JK. 1986. PhD thesis. Cambridge Univ.

Webb JK, Barcons X. 1991. MNRAS 250: 270

Webb JK, Barcons X, Carswell RF, Parnell HC. 1992. MNRAS 255:319

Webb JK, Carswell RF. 1991. In Proc. ESO Mini-Workshop QSO Absorption Lines, ed. PA Shaver, EJ Wampler, AM Wolfe, ESO Sci. Rep. No. 9, p. 3. Garching: Eur. South. Obs.

Webb JK, Larsen I. 1988. In High Redshift and Primeval Galaxies, ed. J Bergeron, D Kunth, B Rocca-Volmerange. Gif-sur-Yvette: Ed. Front.

Weinberg DH, Miralda-Escudé J, Hernquist L, Katz N. 1997. Ap. J. 490:564

Weisheit JC. 1978. Ap. J. 219:829

Weymann RJ, Carswell RF, Smith MG. 1981. Annu. Rev. Astron. Astrophys. 19:41

White SDM. 1979. MNRAS 186:145

Williger GM, Babul A. 1992. Ap. J. 399:385

Williger GM, Baldwin JA, Carswell RF, Cooke AJ, Hazard C, et al. 1994. Ap. J. 428:574

Williger GM, Carswell RF, Webb JK, Boksenberg A, Smith MG. 1989. MNRAS 237:635

Wolfe AM, Turnshek DA, Smith HE, Cohen RD. 1986. Ap. J. 61:249

Womble DS, Sargent WLW, Lyons RS. 1996. In Cold Gas at High Redshift, ed. MN Bremer, et al, p. 137. Dordrecht: Kluwer

Young PJ, Sargent WLW, Boksenberg A. 1982a. Ap. J. Suppl. 48:455

Young PJ, Sargent WLW, Boksenberg A. 1982b. Ap. J. 252:10

Young PJ, Sargent WLW, Boksenberg A, Oke JB. 1981. Ap. J. 249:415

Zeldovich YB. 1970. Astron. Astrophys. 5:84

Zhang Y, Anninos P, Norman ML. 1995. Ap. J. Lett. 453:57

Zhang Y, Anninos P, Norman ML, Meiksin A. 1997. Ap. J. 485:496

Zheng W, Davidsen AF, Kriss GA. 1998. Astron. J. 115:391

Zuo L. 1992. MNRAS 258:45

Zuo L. 1993. Astron. Astrophys. 278:343

Zuo L, Bond JR. 1994. Ap. J. 423:73

Zuo L, Lu L. 1993. Ap. J. 418:601 


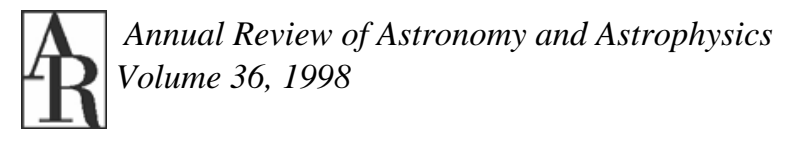

\section{CONTENTS}

Roaming Through Astrophysics, H. C. van de Hulst 1

Type la Supernovae and the Hubble Constant, David Branch 17

Detection of Extrasolar Giant Planets, Geoffrey W. Marcy and R. 57

Paul Butler

First Results from Hipparcos, J. Kovalevsky 99

Radio Emission from Solar Flares, T. S. Bastian, A. O. Benz, and 131

D. E. Gary

Star Formation in Galaxies Along the Hubble Sequence, Robert C. Kennicutt Jr.

189

Herbig Ae/Be Stars, L. B. F. M. Waters, C. Waelkens 233

The Lyman Alpha Forest in the Spectra of Quasistellar Objects, Michael Rauch

Chemical Evolution of Star-Forming Regions, Ewine F. van

Dishoeck and Geoffrey A. Blake

Carbon Stars, George Wallerstein and Gillian R. Knapp 369

Dwarf Galaxies of the Local Group, Mario Mateo 435

Astronomical Searches for Earth-Like Planets and Signs of Life, 507

Neville Woolf and J. Roger Angel

Modeling Extragalactic Jets, Attilio Ferrari 539

Simulations of Structure Formation in the Universe, Edmund 599

Bertschinger 